\title{
Target-space anomalies and elliptic indices in heterotic orbifolds
}

\author{
Claudio A. Scrucca \\ CERN, 1211 Geneva 23, Switzerland \\ E-mail: 'Ćlaudio.'Scruccāocern.ch'

\section{Marco Serone} \\ ISAS-SISSA, Via Beirut 2-4, 34013 Trieste, Italy \\ INFN, sez. di Trieste, Italy \\ E-mail: serone@sissa.it!
}

\begin{abstract}
We present a complete string theory analysis of all mixed gauge, gravitational and target-space anomalies potentially arising in the simplest heterotic $\mathbb{Z}_{N}$ orbifold models, with $N$ odd and standard embedding. These anomalies turn out to be encoded in an elliptic index, which can be easily computed; they are found to cancel through a universal GS mechanism induced by the dilaton multiplet. The target-space symmetry is then shown to have a nice geometric interpretation in terms of torsion, and the target-space dependence of the four-dimensional GS couplings can be alternatively rederived from the implicit torsion dependence of the standard ten-dimensional GS couplings. The result is universal and consists essentially of a Bianchi identity for the NSNS $B$ field depending on all the curvatures, and in particular on the target-space curvature.
\end{abstract}

KEYwords: Superstrings and Heterōtic Strings, Anomalies in Field and Stringi Theörièsi 


\section{Contents}

ii. Introduction

2. Anomalies in heterotic string theory

3. Orbifolds and elliptic indices

is. Field theory anomaly

13.2 GS terms

44. Low-energy interpretation

5. Geometric structure and relation to torsion

6. Conclusions

'Aي.'. Characteristic classes

블." Decomposition of characters

C.:- Limits of the partition functions

'D- Chiral determinants

\section{Introduction}

Target-space duality symmetries (see [i] and references therein) in orbifold compactifications of heterotic string theory [2: have received a lot of attention in the past and have recently been the object of renewed interest [is] heterotic-type-I duality [8] $D=4$ heterotic and type-IIB orientifold vacua [9-10 - At the level of the lowenergy supergravity effective action, these symmetries correspond typically to global isometries acting on the manifold describing the moduli space of scalar fields. An example is given by $D=4 N=1$ heterotic orbifold models, which admit $\operatorname{SL}(2, \mathbb{R})_{i}$ target-space duality symmetries acting in a peculiar way on the untwisted moduli $T_{i}$ that describe the Kähler structure of the internal space 14 . Such symmetries are 


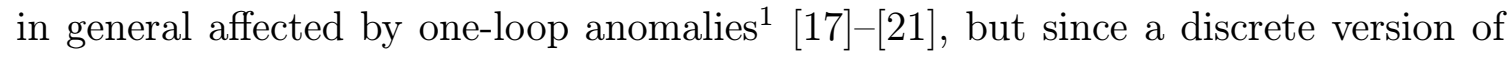
them (T-duality) is believed to be an exact symmetry of heterotic string theory, the latter are expected to cancel by some mechanism.

Although anomalies in target-space duality symmetries (in the following denoted simply target-space anomalies) can arise in different channels, most of the attention in the past has been devoted to mixed target-space/gauge and targetspace/gravitational anomalies for their relation to threshold corrections. It was realized that such field theory one-loop anomalies can be generically cancelled by the

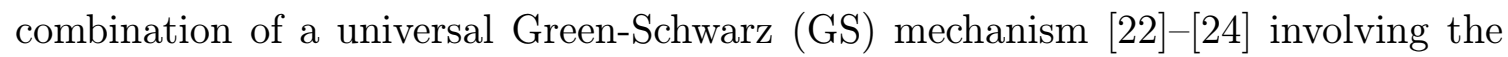
dilaton multiplet and the anomalous variation of threshold corrections to gauge cou-

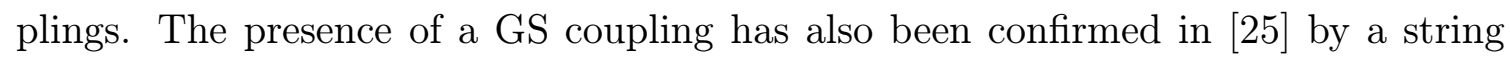
computation. Other cases, such as purely target-space anomalies, have received little attention to date, and as far as we know, have been analysed only in [2] $\overline{2} \overline{1}_{1}$, from a low-energy field theory point of view. It should be noticed that in all previous analyses, target-space anomalies have always been computed indirectly, by exploiting their similarity with gauge anomalies. The difficulty of a direct computation resides in the fact that the connection associated to a target-space duality symmetry is a composite rather than an elementary field.

In this paper, we reconsider this issue through an explicit string theory computation of all possible target-space/gauge/gravitational anomalies in heterotic orbifold models. We restrict to models without threshold corrections, where anomalies are expected to be cancelled through a GS mechanism only, and focus on the simplest $\mathbb{Z}_{N}$ models with $N$ odd and standard embedding, i.e. the $\mathbb{Z}_{3}$ and the $\mathbb{Z}_{7}$ models. Anomalous amplitudes in heterotic string theory have been extensively studied in the past. In this work, we proceed along the lines of $[\underline{2} 6]-[\underline{2}-2]$ and $[2 \underline{2}]-[\underline{3} 2]$ to identify the generalizations of the elliptic genus [3. under consideration. By evaluating both the modular-invariant and holomorphic versions of the elliptic genus, we are then able to derive both the one-loop field theory anomaly and the GS couplings. We show that the one-loop anomaly nicely factorizes and is completely cancelled by a GS mechanism. In a low-energy description in terms of chiral multiplets, the latter is induced by an inhomogeneous transformation of the dilaton multiplet, and the cancellation of pure target-space anomalies requires an appropriate kinetic term for the composite target-space connection in the low-energy

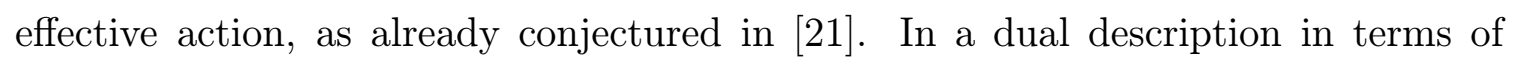
linear multiplets, this term modifies the Bianchi identity for the NSNS two-form $B$ dual to the universal axion by a source term involving the target-space curvature.

The results we find agree with those derived in $[1 \overline{1} \overline{9}, 1,20]$ target-space/gravitational anomalies, and with the various modular weights assign-

\footnotetext{
${ }^{1}$ The presence of anomalies in this kind of symmetries was first addressed in [1] possible cancellation through a generalized GS mechanism noted in [1] $\left.\mathbf{1}_{1}^{6}\right]$.
} 


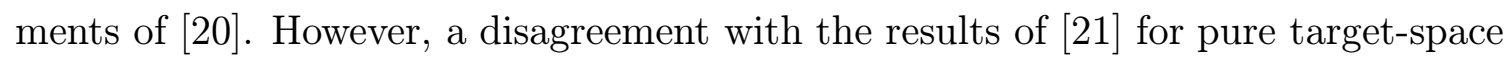
anomalies is found. This suggests that the analogy between target-space and gauge anomalies used in [21] could present subtleties related to the compositeness of the target-space connection, and might be incorrect when more than one composite connection occurs as external states. In this respect, we have checked that the anomaly computed along the lines of [i페] does not factorize and hence could not be cancelled by a GS mechanism.

We also clarify the geometrical structure of target-space anomalies by showing that the four-dimensional target-space curvatures are nothing but the internal components of the ten-dimensional torsion-full curvature two-form. The additional term in the Bianchi identity for $B$ can then be deduced from the implicit torsion

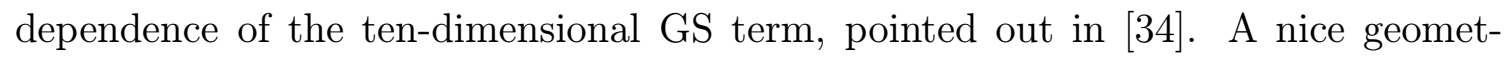
ric interpretation can also be made of the contribution of each single state to the anomaly in our string set-up. In particular, target-space anomalies resemble standard gravitational and gauge anomalies in the untwisted and twisted sectors of the orbifold, respectively. This is explained by the quite different dependence on the internal metric and volume in the two cases.

The structure of this paper is as follows. In section 2 , we review well-known general properties of anomalous heterotic amplitudes. In section we describe our strategy for the string computation and hence compute the elliptic genus relevant to all target-space/gauge/gravitational anomalies. Some comments on the field-theory interpretation of our results are then given in section 's.', whereas the geometric structure of these anomalies, including their relation with torsion, is given in section Finally, section ${ }_{-1}^{6}$ i contains some conclusions. In addition, we report some conventions

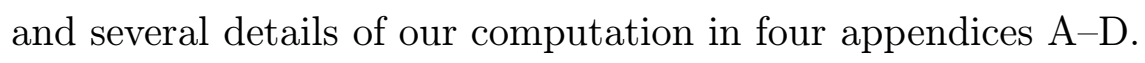

\section{Anomalies in heterotic string theory}

In string theory, anomalous amplitudes happen to be total modular derivatives, and therefore receives contributions only from the boundaries of the moduli space of the relevant world-sheet surface. In heterotic models, for instance, anomalies are expressed as integrals over the boundary $\partial \mathcal{F}$ of the fundamental domain $\mathcal{F}$ of toroidal world-sheets, and their cancellation is then a direct consequence of modular invari-

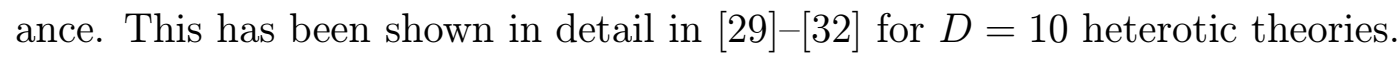

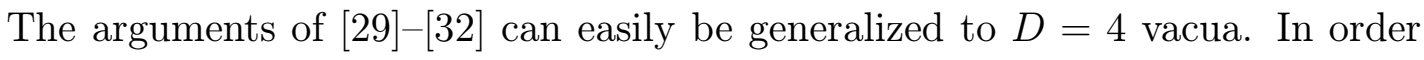
to do so, it is convenient to regard the anomaly as a possible non-vanishing amplitude involving an unphysical longitudinally polarized particle. The relevant string world-sheet is a torus. Moreover, since standard chiral anomalies only arise in the CP-odd part of the effective action, whereas others, such as target-space anomalies, distribute in a supersymmetric way between CP-even and CP-odd parts, it will be sufficient to restrict to correlations in the odd spin structure. Recall that in this 
completely periodic spin structure there is a world-sheet gravitino zero-mode inducing the insertion of a world-sheet supercurrent, the picture-changing operator $T_{F}$. Moreover, owing to the presence of a Killing spinor, one of the vertex operators must be taken in the so-called (-1)-picture, while all the others can be taken in the 0 -picture [35. It is easy to verify that anomalous amplitudes can arise only when the vertex operator associated to the longitudinal particle is the one in the

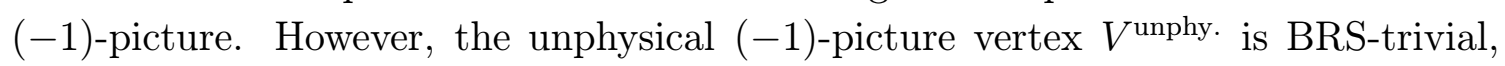

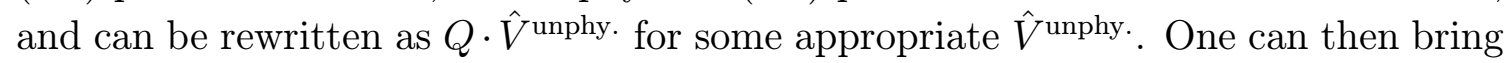
$Q$ to act on the rest of the correlation. Since the action of $Q$ on the physical 0 -

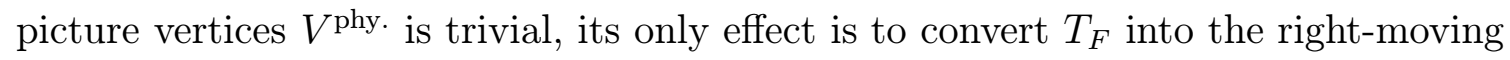
world-sheet energy-momentum tensor $T_{B}: Q \cdot T_{F}=T_{B}$. Finally, the net effect of the insertion of $T_{B}$ is to produce the derivative with respect to the torus modulus $\bar{\tau}$ of the remaining correlation. ${ }^{2}$ More precisely, one finds as expected a total derivative in moduli space. A generic anomalous amplitude is therefore of the form $\mathcal{A}=\oint_{\partial \mathcal{F}} d \tau\left\langle\hat{V}_{1}^{\text {unphy. }} V_{2}^{\text {phy. }} \cdots V_{n}^{\text {phy. }}\right\rangle$, the relevant number of vertex operators being determined by the integration over the Grassmann fermionic zero modes. Importantly, such an anomaly $\mathcal{A}$ satisfies the Wess-Zumino (WZ) consistency condition, and is the WZ descent of $I_{\mathcal{A}}=\oint_{\partial \mathcal{F}} d \tau\left\langle V_{1}^{\text {phy. }} V_{2}^{\text {phy. }} \cdots V_{n}^{\text {phy. }}\right\rangle: \mathcal{A}=2 \pi i I_{\mathcal{A}}^{(1)}{ }^{3}$ The total anomaly polynomial $I$ encoding all the anomalous amplitudes of this type is then given by

$$
I=\oint_{\partial \mathcal{F}} d \tau \bar{A}(\tau)
$$

where $\bar{A}(\tau)$ is the generating functional of odd spin structure correlation functions, i.e. the partition function computed by deforming the free action with the vertex operators appearing in anomalous amplitudes. The result ( $\left.\overline{2}_{-} . \overline{1_{1}}\right)$ is completely general and its WZ descent produces the full set of anomalies. The integration over fermionic zero modes in $\bar{A}(\tau)$ automatically selects the components of appropriate degree in any space-time dimensionality.

From a string theory point of view, the vanishing of the total anomaly is a

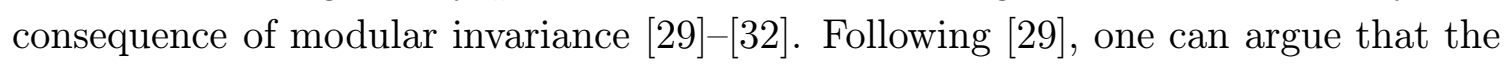
contribution from the part of $\partial \mathcal{F}$ closing at infinity vanishes by analytic continuation from kinematical regions where the correlation of the factors $\exp i p \cdot X$ in the vertex operators give an exponential suppression. The rest of the integral over $\partial \mathcal{F}$ vanishes instead under the condition that the generating functional $\bar{A}$ is modular-invariant:

$$
\begin{aligned}
& \bar{A}(\tau+1)=\bar{A}(\tau), \\
& \bar{A}(-1 / \tau)=\tau^{2} \bar{A}(\tau) .
\end{aligned}
$$

\footnotetext{
${ }^{2}$ For convenience, we take $\tau \leftrightarrow \bar{\tau}$ with respect to the standard notation for the heterotic string.

${ }^{3}$ We use here the standard WZ descent notation. Given a gauge-invariant $D+2$ form $I$, one can define a $D+1$ Chern-Simons form $I^{(0)}$ such that $I=d I^{(0)}$, whose variation is exact and defines a $D$-form $I^{(1)}$ through $\delta I^{(0)}=d I^{(1)}$.
} 
This can be easily understood from figure ${ }_{-1}^{1}$ Indeed, the contributions from parts I

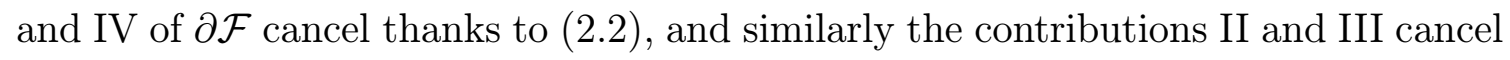
by virtue of $(\underline{2} . \overline{3})$. Finally, one finds therefore:

$$
I=0
$$

At the level of low-energy effective action, it is well-known that (12.4') appears as a cancellation between a one-loop anomaly and a tree-level Green-Schwarz inflow.

These two contributions can be explicitly identified in the low-energy limit $\alpha^{\prime} \rightarrow$ 0 of the above string theory computation. In fact, it was shown in $\left[\overline{2} \bar{g} \bar{g}_{1}\right]$ that the lowenergy limit of $\bar{A}(\tau)$ is technically equivalent to its $\tau \rightarrow i \infty$ limit. Using this result, it is possible to disentangle the contribution corresponding to the one-loop anomaly from the contribution of the GS inflow, which is seen to factorize on a pole corresponding to the exchange of the NSNS two-form $B$.

The low-energy interpretation can be made more concrete by explicitly evaluating the generating functional $\bar{A}$ at leading order in $\alpha^{\prime} \rightarrow 0$. In this limit, the path integral representation can be evaluated ex-

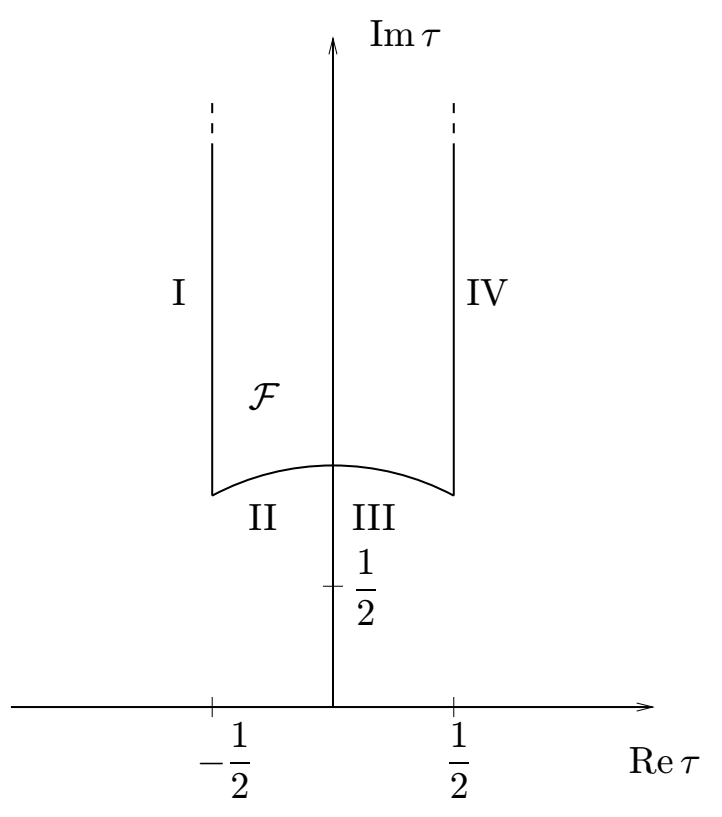

Figure 1: Fundamental domain of the torus. actly, using a modular-invariant regularization. It becomes clear that $\bar{A}$ is intimately related to a character-valued index $A$, obtained from the same path-integral but with a holomorphic regularization. This is

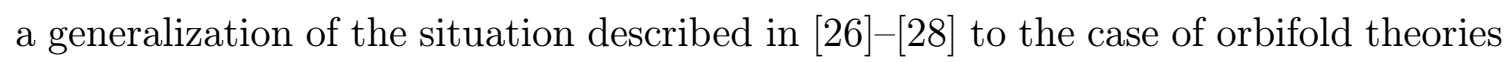
with an additional target-space background. With a slight abuse of language, we will simply refer to $A$ and $\bar{A}$ as elliptic genera in the following. On general grounds, the modular-invariant elliptic genus $\bar{A}$ differs from the holomorphic elliptic genus $A$ only by a so-called modular anomaly involving some four-form $X_{4}\left[\begin{array}{l}{[\overline{2}} \\ \overline{Z_{1}}\end{array}\right]$. The precise relation is

$$
\bar{A}(\tau)=\exp \left\{-\frac{X_{4}}{64 \pi^{3} \operatorname{Im} \tau}\right\} A(\tau),
$$

where $X_{4}$ is in fact entirely determined by the requirement of modular invariance, and can be computed in a direct way using a specific modular-invariant regularization prescription (see appendix 'D-1's).

The appearance of the modular-invariant version $\bar{A}$ of the elliptic genus $A$ in the expression for the anomaly polynomial is of course not a coincidence. Indeed, it is well known that its holomorphic companion $A$ represents the chiral index of the full 
string spectrum, and the one-loop field-theory anomaly associated to chiral massless states is therefore given by $\left[\overline{2} \overline{\sigma_{1}}\right]$

$$
I_{F T}=\lim _{\tau \rightarrow i \infty} A(\tau)=X_{6}
$$

where the second equality anticipates that only the six-form component of the result is relevant in $D=4$. The GS term, in turn, can be obtained by generalizing the work of [2] genus $\bar{A}$ and is given by

$$
L_{G S}=B \wedge\left[\frac{1}{64 \pi^{2}} \int_{\mathcal{F}} \frac{d^{2} \tau}{(\operatorname{Im} \tau)^{2}} \bar{A}(\tau)\right],
$$

where $B$ is the NSNS two-form, dual to the axion in four dimensions. Using then the general expression ( $\left(\overline{2}_{2} . \overline{5}_{1}\right)$ into ( $\left(\overline{2} . \overline{7}_{1}\right)$, and going through the same manipulations as in $[\overline{2} \bar{z} \bar{z}]$, the modular integral yielding the GS term can easily be evaluated. In $D=4$, one finds

$$
L_{G S}=-2 \pi B \wedge X_{2},
$$

where the two-form $X_{2}$ is formally defined as $X_{2}=X_{6} / X_{4}$. Clearly, this makes sense only if $X_{6}=X_{2} \wedge X_{4}$, i.e. only if the field theory anomaly factorizes. This must be guaranteed by the form (12.5. induced inflow of anomaly is $I_{G S}=-X_{6}$ if and only if the Bianchi identity satisfied by the field strength $H$ of the $B$ field is

$$
d H=X_{4} .
$$

Summarizing, the vanishing of the total string theory anomaly is interpreted at low energies as the cancellation of the field-theory anomaly through the GS mecha-

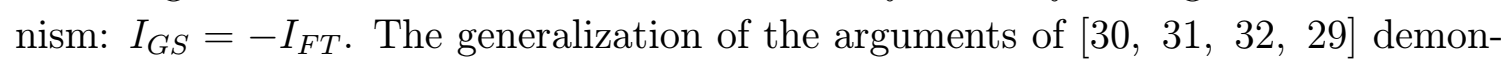
strates that anomaly cancellation at the string-theory level is a consequence of modular invariance, whereas the generalization of the arguments of $[\overline{2} \overline{2} \overline{1}, 1,12 \overline{2} \overline{1}]$ allows us to interpret the field-theory counterpart of the cancellation mechanism. Intuitively, one can think of the field-theory anomaly as the $A$ part of $\bar{A}$, and attribute the GS inflow to the anomalous phase through which $\bar{A}$ differs from $A$.

As a remark, we would like to propose a very naive but suggestive alternative way to understand how the vanishing of the anomaly is achieved at the string-theory level. It is motivated by the unpleasant feature that the above arguments involve killing the contribution from infinity in $\partial \mathcal{F}$ by analytic continuation, whereas (2. $2 . \overline{6})$ suggests that this contribution should instead be directly linked to the field-theory anomaly. The point is that the field-theory Schwinger parameter associated to the modulus $\tau$ is actually $\alpha^{\prime} \tau$, so that the various contributions to the anomaly appear from potentially different corners of moduli space, depending on whether one takes $\alpha^{\prime} \rightarrow 0$ from the beginning or only at the end. This is also the essence of the 
already mentioned observation of [129i] about the technical equivalence of the two limits $\alpha^{\prime} \rightarrow 0$ and $\tau \rightarrow i \infty$ in anomalous amplitudes. In particular, if one takes brutally $\alpha^{\prime} \rightarrow 0$ from the beginning, one can no longer kill the contribution at infinity in $\partial \mathcal{F}$ by analytic continuation, since each pair of momenta is accompanied by an $\alpha^{\prime}$.

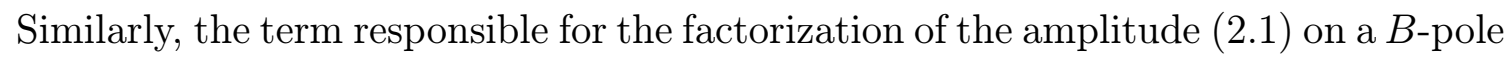
is suppressed. These two effects seem to compensate each other, and as a matter of fact, one could recover a natural interpretation of the cancellation mechanism by taking the $\alpha^{\prime} \rightarrow 0$ from the beginning and computing the elliptic genus with a

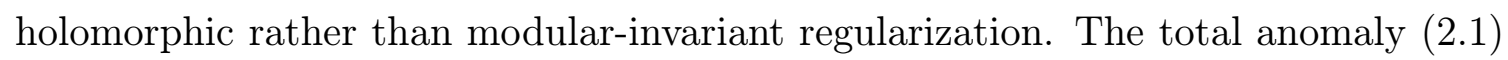
would then involve $A$ instead of $\bar{A}$, and the vanishing of the contour integral would be attributed to holomorphicity of $A$ in $\mathcal{F}$, rather than modular invariance. However, the relevant pieces of $\partial \mathcal{F}$ are now different. The contribution at infinity yields the field-theory anomaly. The contributions from I and IV still cancel, because (2.2) is still satisfied for $A$. The contributions from II and III, instead, no longer cancel since (2.3in $)$ fails for $A$, but rather combine to yield the GS inflow. Although this alternative argument cannot be taken too seriously, we find it quite suggestive.

\section{Orbifolds and elliptic indices}

In this section, we shall provide a concrete example of the general features discussed in the previous section, by studying all possible gauge/gravitational/target-space anomalies for $\mathbb{Z}_{N}$ orbifold models with standard embedding and $N$ odd, i.e. the $\mathbb{Z}_{3}$ and $\mathbb{Z}_{7}$ models. According to the arguments above, this involves computing the elliptic genus in a gauge, gravitational and target-space background, in a sense that we shall now make more precise.

To begin with, let us briefly recall some basic facts about $\mathbb{Z}_{N}$ heterotic orbifolds. The generator of the orbifold action is defined by a twist vector $v_{i}$, where $i=1,2,3$ label the three internal tori and the associated complex coordinates, satisfying the condition $\sum_{i} v_{i}=0$. For standard embedding, the orbifold action on the gauge lattice is simply a shift by $v_{i}$ itself and acts on an $\mathrm{SU}(3)$ part of the Cartan subgroup $\mathrm{SO}(16)$ of one of the two $E_{8}$ factors $\left(v_{j}=0\right.$ for $\left.j>3\right)$. The total gauge group is then $E_{8} \times E_{6} \times H$, where $H$ is a subgroup of $\mathrm{SU}(3)$ (see table $\left.\mathbf{i}_{1}\right)$.

It is well known that these heterotic vacua possess a target-space duality symmetry. This symmetry acts as $\operatorname{SL}(2, \mathbb{R})_{i}$ transformations on the three universal diagonal Kähler moduli superfields $T_{i}$ [i]

$$
T_{i} \rightarrow \frac{a_{i} T_{i}-i b_{i}}{i c_{i} T_{i}+d_{i}}
$$

Its discrete $\mathrm{SL}(2, \mathbb{Z})$ subgroup is an exact symmetry (to all orders in $\alpha^{\prime}$ and $g_{s}$ ) of

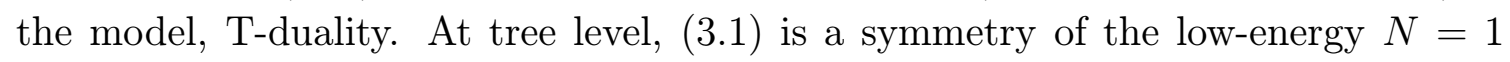


supergravity effective action, provided one transforms in an appropriate way each matter chiral superfield as well, requiring:

$$
\Phi_{s} \rightarrow \exp \left\{-n_{i} \ln \left(i c_{i} T_{i}+d_{i}\right)\right\} \Phi_{s}
$$

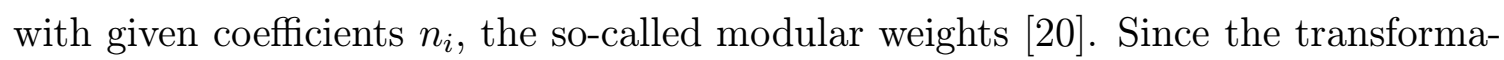

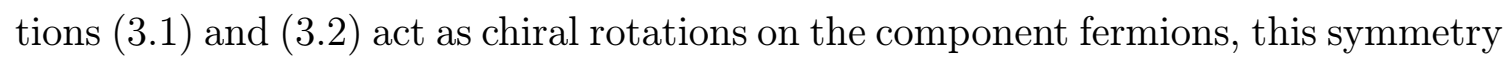
is potentially anomalous. At one loop, anomalies appear through triangular graphs

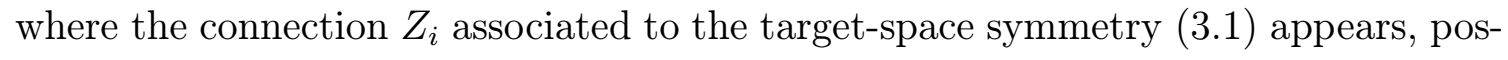

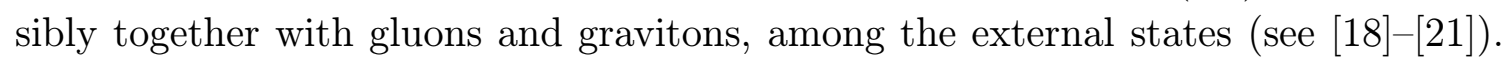
Although such anomalies (mainly mixed ones) have received quite a lot of attention in the past, no direct, explicit and complete computation of them has so far appeared in the literature. A satisfactory understanding of their structure is also missing. Since the target-space connections $Z_{i}$ are composite and not elementary fields (see ( $\left(\hat{3}_{3} . \overline{1} \overline{6}_{i}^{i}\right)$ ), such anomalies are typically derived by analogy with standard gauge anomalies associated to an elementary connection. This approach was adopted also in [2]-1], the only reference considering target-space/gauge/gravitational anomalies in full generality.

The approach we follow here is more direct: we will compute anomalous amplitudes involving the elementary constituents of the composite connections $Z_{i}$. We focus on the dependence on the diagonal untwisted moduli. These are defined as:

$$
T_{i}=G_{i \bar{\imath}}+i B_{i \bar{\imath}}
$$

where the scalars $G_{i \bar{\imath}}$ and the pseudoscalars $B_{i \bar{\imath}}$ represent the internal metric and $B$-field components of the orbifold in complex coordinates along each of the three $T_{i}^{2}$ tori. It is straightforward to determine the most general form of the targetspace connections $Z_{i}$ and their associated field strengths $G_{i}$ in terms of these fields. The total connection one-form is $Z=\sum_{i}\left(\alpha_{i} d T_{i}+\bar{\alpha}_{\bar{\imath}} d \bar{T}_{\bar{\imath}}\right)$, where the $\alpha_{i}$ 's are some functions of the $T$-moduli. Correspondingly, the general form of the field strength $G=d Z$ is

$$
G=\frac{1}{2} \sum_{i, j}\left[\alpha_{i, j} d T_{i} \wedge d T_{j}+\bar{\alpha}_{\bar{\imath}, \bar{\jmath}} d \bar{T}_{\bar{\imath}} \wedge d \bar{T}_{\bar{\jmath}}+\left(\alpha_{i, \bar{\jmath}}-\bar{\alpha}_{\bar{\jmath}, i}\right) d T_{i} \wedge d \bar{T}_{\bar{\jmath}}\right] .
$$

From (3.4i $)$, it is clear that the composite field strength is always at least quadratic in the fluctuations of the moduli ( anomalous triangular graphs with external composite fields by replacing each of them with a couple of $T$ fields. Actually, it is known from supergravity that the coefficients $\alpha_{i}$ are determined by the Kähler potential $K$ as $\alpha_{i}=-i \partial K / \partial T_{i}=-i K_{i}{ }^{4}$ The field

\footnotetext{
${ }^{4}$ The covariant derivative of a generic chiral multiplet contains the sum of the Kähler and the $\sigma$-model connections, proportional to each other through the modular weight. The target-space connection is identified with the Kähler connection, and the total charge of each state is $1+2 n_{i}$.
} 
strength (…4.

$$
G=-i \sum_{i, j} K_{i, \bar{\jmath}} d T_{i} \wedge d \bar{T}_{\bar{\jmath}}
$$

Finally, the relevant part of the Kähler potential for the models under consideration is $K=-\sum_{i} \ln \left(T_{i}+\bar{T}_{\bar{\imath}}\right)$, yielding a diagonal result: $K_{i, \bar{\jmath}}=\delta_{i, \bar{\jmath}} / 2\left(T_{i}+\bar{T}_{\bar{\imath}}\right)^{2}$. In the following, we shall assume only the general form ( $\left(\overline{3}_{-1} \overline{4}_{1}\right)$, and verify that the supergravity result $(\underline{13} . \overline{5}-\overline{1})$ is correctly recovered.

In order to evaluate the elliptic genus as a function of the gauge, gravitational and target-space backgrounds, we need to analyse the corresponding gluon, graviton and moduli vertex operators. According to the general discussion of section we have to take into account only physical operators in the 0-picture. Consider first the standard case of photons and gravitons. Denoting with $J^{a}$ the current operator of the gauge current algebra, and with $Q_{a}$ its zero mode, one has:

$$
\begin{aligned}
& V_{g}=\xi_{\mu \nu}(p) \int d^{2} z \partial X^{\mu}(z)\left(\bar{\partial} X^{\nu}+i p \cdot \psi \psi^{\mu}\right)(\bar{z}) e^{i p \cdot X(z, \bar{z})}, \\
& V_{\gamma}=\xi_{\mu}^{a}(p) \int d^{2} z J^{a}(z)\left(\bar{\partial} X^{\mu}+i p \cdot \psi \psi^{\mu}\right)(\bar{z}) e^{i p \cdot X(z, \bar{z})} .
\end{aligned}
$$

In the low-energy limit $\alpha^{\prime} \rightarrow 0$, one can restrict to the terms providing a minimal number of momenta and a maximal number of fermionic zero-modes, and one finds:

$$
\begin{aligned}
& V_{g}^{\text {eff. }}(R)=R_{\mu \nu} \int d^{2} z X^{\mu}(z) \partial X^{\nu}(z), \\
& V_{\gamma}^{\text {eff. }}(F)=F^{a} Q^{a},
\end{aligned}
$$

where $F^{a}$ and $R_{\mu \nu}$ represent the gauge and gravitational curvatures and are given by

$$
F^{a}=\frac{1}{2} F_{\mu \nu}^{a} \psi_{0}^{\mu} \psi_{0}^{\nu}, \quad R_{\mu \nu}=\frac{1}{2} R_{\mu \nu \rho \sigma} \psi_{0}^{\rho} \psi_{0}^{\sigma} .
$$

Consider next the case of the $T_{i}$ moduli. Their vertex operators are given by: ${ }^{5}$

$$
\begin{aligned}
& V_{T_{i}}=T_{i}(p) \int d^{2} z \partial \bar{X}^{\bar{c}}(z)\left(\bar{\partial} X^{i}+i p \cdot \psi \psi^{i}\right)(\bar{z}) e^{i p \cdot X(z, \bar{z})} \\
& V_{\bar{T}_{\bar{\imath}}}=\bar{T}_{\bar{\imath}}(\bar{p}) \int d^{2} z \partial X^{i}(z)\left(\bar{\partial}^{\bar{\imath}}+i \bar{p} \cdot \psi \bar{\psi}^{\bar{\imath}}\right)(\bar{z}) e^{i \bar{p} \cdot X(z, \bar{z})}
\end{aligned}
$$

and lead to:

$$
\begin{aligned}
& V_{T_{i}}^{\text {eff. }}=d T_{i} \int d^{2} z \psi^{i}(\bar{z}) \partial \bar{X}^{\bar{\imath}}(z), \\
& V_{\bar{T}_{\bar{\imath}}}^{\text {eff. }}=d \bar{T}_{\bar{\imath}} \int d^{2} z \bar{\psi}^{\bar{\imath}}(\bar{z}) \partial X^{i}(z),
\end{aligned}
$$

\footnotetext{
${ }^{5} \operatorname{In}\left(\bar{p}_{3} . \overline{1} \bar{q}\right), \bar{p}$ denotes simply the momentum of $\bar{T}$, not the complex conjugate of $p$.
} 
where

$$
d T_{i}=i p_{\mu} T_{i} \psi_{0}^{\mu}, \quad d \bar{T}_{\bar{\imath}}=i \bar{p}_{\mu} \bar{T}_{\bar{\imath}} \psi_{0}^{\mu} .
$$

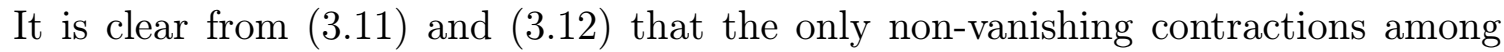
moduli occur between a given $T_{i}$ modulus and its complex conjugate $\bar{T}_{\bar{\imath}}$. A generic correlation is therefore non-vanishing only if it includes an equal number of $T_{i}$ and $\bar{T}_{\bar{\imath}}$ vertices. This general property can be easily understood also in the path-integral representation of the generating functional. Indeed, integrating out the internal fermions appearing in $\left(\overline{3}, \overline{1} \overline{1}_{1}^{1}\right)$ and rescaling the internal bosonic fields to normalize their kinetic terms, one ends up with an effective bosonic interaction that is interpreted as the effective vertex operator for the composite connection. This manipulation is equivalent to grouping in all possible ways the moduli vertices in $T_{i}-\bar{T}_{\bar{\imath}}$ pairs and explicitly performing the fermionic contraction within each pair. One finds:

$$
V_{Z_{i}}^{\mathrm{eff} .}\left(G_{i}\right)=G_{i} \int d^{2} z \bar{X}^{\bar{\imath}}(z) \partial X^{i}(z)
$$

with $G_{i}$ representing the target-space curvature two-forms and given by

$$
G_{i}=\frac{i}{2\left(T_{i}+\bar{T}_{\bar{\imath}}\right)^{2}} p_{\mu} T_{i} \bar{p}_{\nu} \bar{T}_{\bar{\imath}} \psi_{0}^{\mu} \psi_{0}^{\nu}
$$

As expected, the external moduli automatically group in $T_{i} \bar{T}_{\bar{\imath}}$ pairs, reconstructing compos-

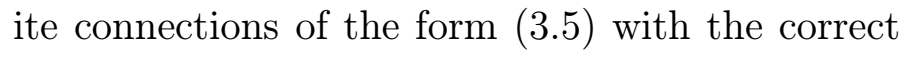
Kähler potential. Correpondingly, one can define three independent connections and curvatures associated to each internal torus and given by

$$
\begin{aligned}
Z_{i} & =\frac{-i}{\left(T_{i}+\bar{T}_{\bar{\imath}}\right)} d\left(T_{i}-\bar{T}_{\bar{\imath}}\right), \\
G_{i} & =\frac{-i}{2\left(T_{i}+\bar{T}_{\bar{\imath}}\right)^{2}} d T_{i} \wedge d \bar{T}_{\bar{\imath}} .
\end{aligned}
$$

It is important to stress that within this direct approach, the pairing of the moduli appearing as external states into composite connections is a de-

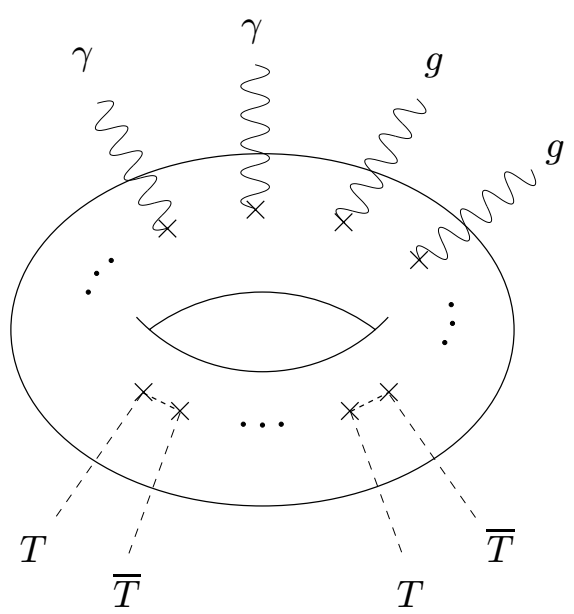

Figure 2: Pairing of moduli in anomalous amplitudes. rived property and not an assumption. This mechanism is depicted in figure $12 \overline{2}$ for a generic anomalous amplitude.

The computation of the elliptic genus does not present any problem, since all the effective vertices are quadratic. We begin with the holomorphic regularization. The result factorizes into space-time, compact and gauge partition functions:

$$
A(\tau ; F, R, G)=\frac{1}{2 N} \sum_{k, l=0}^{N-1} N_{k, l} Z_{S T}(R) Z_{C}^{k, l}(G) Z_{\Gamma}^{k, l}(F)
$$


The $1 / 2 N$ factor is due to the GSO projection in a $\mathbb{Z}_{N}$ orbifold model and $N_{k, l}$ represents the number of points that are at the same time $k$ - and $l$-fixed. Restricting to the six-form component of the elliptic genus relevant in four dimensions, one can rescale the free-particle normalization to cancel the $\tau$-dependence coming from the curvatures. The only $\tau$ dependence that is left over arises then through $q=e^{2 i \pi \tau}$. For $(k, l) \neq(0,0)$, one finds the following results in terms of the skew eigenvalues $y_{i}, \lambda_{a}$ and $g_{i}$ of the rescaled gauge, gravitational and target space curvatures (see appendix $\underline{A}_{\text {A }}$ ) (recall that $v_{j}=0$ for $\left.j>3\right):^{6}$

$$
\begin{aligned}
& Z_{S T}(R)=\eta^{2}(\tau) \prod_{a=1}^{2} \frac{\left(i \lambda_{a}\right) \eta(\tau)}{\theta_{1}\left(i \lambda_{a} / 2 \pi \mid \tau\right)} \\
& Z_{C}^{k, l}(G)=\prod_{i=1}^{3} \frac{\eta(\tau)}{\theta_{1}\left[\begin{array}{l}
l v_{i} \\
k v_{i}
\end{array}\right]\left(-g_{i} / 2 \pi \mid \tau\right)}, \\
& Z_{\Gamma}^{k, l}(F)=\frac{1}{4} \sum_{\alpha, \beta} \prod_{i=1}^{8} \frac{\theta_{\alpha}\left[\begin{array}{l}
l v_{i} \\
k v_{i}
\end{array}\right]\left(-y_{i} / 2 \pi \mid \tau\right)}{\eta(\tau)} \prod_{h=1}^{8} \frac{\theta_{\beta}\left(-y_{h}^{\prime} / 2 \pi \mid \tau\right)}{\eta(\tau)}
\end{aligned}
$$

In the completely untwisted sector $(k, l)=(0,0)$, there are six additional fermionic zero modes arising from the compact dimensions, and the result is apparently vanishing. Actually, there is a potential contribution from this sector to pure target-space anomalies of the form $G_{1} G_{2} G_{3}$. This can be easily understood by noting from ( $\left.\overline{3} . \overline{1} \overline{1}_{1}^{1}\right)$

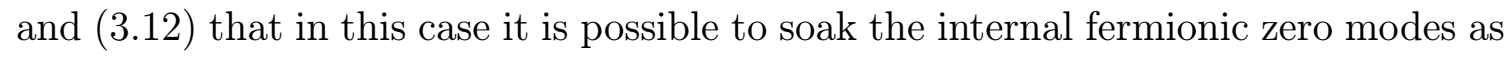
well. However, the correlation that one obtains involves only internal right-moving momenta, and is clearly irrelevant in the limit $\left(\underline{2} . \overline{6}_{-}^{\prime}\right)$ and for the exponential term in $\left(2.5_{1}\right)$. Hence, it will be completely neglected in the following. ${ }^{7}$

The corresponding result in the modular-invariant regularization summarized in

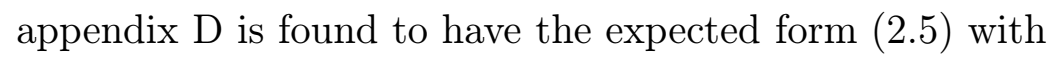

$$
X_{4}(F, R, G)=8 \pi^{2}\left(\sum_{a=1}^{2} \lambda_{a}^{2}+\sum_{i=1}^{3} g_{i}^{2}-\sum_{i=1}^{8} y_{i}^{2}-\sum_{h=1}^{8} y_{h}^{\prime 2}\right) .
$$

This is just what is needed for $\bar{A}$ to satisfy the modular transformation proper-

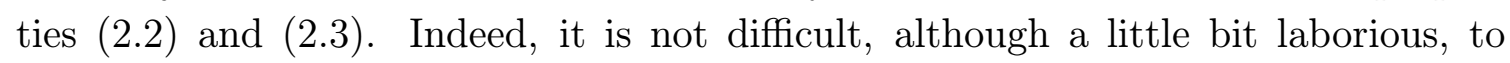
verify that:

$$
\begin{aligned}
& \bar{A}(\tau+1 ; F, R, G)=\bar{A}(\tau ; F, R, G) \\
& \bar{A}\left(-\frac{1}{\tau} ; F, R, G\right)=\frac{1}{\tau} \bar{A}(\tau ; F \tau, R \tau, G \tau)=\tau^{2} \bar{A}(\tau ; F, R, G)
\end{aligned}
$$

where the last step in the second equation holds for the relevant six-form component.

\footnotetext{
${ }^{6}$ The definition of twisted $\theta$-functions used here is taken from $\left[\overline{7}_{1}\right.$, appendix A].

${ }^{7}$ From a more physical point of view, it is clear that the completely untwisted $(0,0)$ sector, having $N=4$ supersymmetry, cannot give rise to any anomaly.
} 
Before entering into the details of the expansion of eqs. $\left(b_{3} . \overline{1} \overline{9}_{1}\right)-\left({ }_{3} .2 \overline{1}_{1}\right)$, it is convenient to define the quantity

$$
C_{k}=\prod_{i=1}^{3} \sin \pi k v_{i},
$$

and the representative

$$
\theta_{i}^{l}=l v_{i}-\operatorname{int}\left(l v_{i}\right)
$$

of the generic twist vector $l v_{i}$ in the interval $[0,1]$. Whereas $\sum_{i} v_{i}$ is 0 in order to have $N=1$ supersymmetry, $\sum_{i} \theta_{i}^{l}$ is 1 for half of the twisted sectors and 2 for the conjugate half. More precisely, one can define the sign $\epsilon_{l}$ distinguishing between conjugate twisted sectors as

$$
\epsilon_{l}=-(-1)^{\sum_{i} \theta_{i}^{l}}=-\operatorname{sign}\left(C_{l}\right)
$$

We also define $S_{l}$ to be the set of twisted sectors where $\epsilon_{l}=1$. The set $S_{l}$ is $\{1\}$ for $\mathbb{Z}_{3}$ and $\{1,2,4\}$ for $\mathbb{Z}_{7}$.

\subsection{Field theory anomaly}

The field theory anomaly $I_{F T}$ can now be computed according to $\left(\mathfrak{n}_{2} . \overline{6}_{6}^{\prime}\right)$. Taking the limit $\tau \rightarrow i \infty$ of $A$ turns out to be a rather non-trivial exercise. For this reason, we

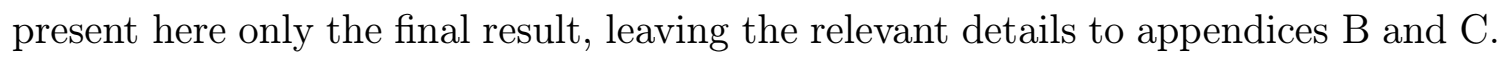
As expected, simple characteristic classes, defined in appendix Since the visible gauge group is $E_{6} \times H$, with $H \subset \mathrm{SU}(3)$ depending on the orbifold projection, it is useful to refer to the underlying $E_{6} \times \mathrm{SU}(3)$ group common to all orbifold models with standard embedding. In the untwisted sector, one finds

$$
\begin{aligned}
I_{F T}^{\text {untw. }}(F, R, G)=\frac{1}{2 N} \sum_{k=1}^{N-1} C_{k}[ & \widehat{A}_{k}(G) \widehat{G}(R)+\widehat{G}_{k}(G) \widehat{A}(R)+ \\
& +\left(\operatorname{ch}_{\mathbf{2 4 8}}(F)+\operatorname{ch}_{(\mathbf{7 8 , 1})}(F)+\operatorname{ch}_{(\mathbf{1}, \mathbf{8})}^{k}(F)+\right. \\
& \left.\left.\quad+\operatorname{ch}_{(\mathbf{2 7}, \mathbf{3})}^{k}(F)+\operatorname{ch}_{(\overline{\mathbf{2 7}, \overline{\mathbf{3}})}}^{k}(F)\right) \widehat{A}_{k}(G) \widehat{A}(R)\right]
\end{aligned}
$$

in terms of the twisted Chern characters $\operatorname{ch}_{\rho}^{k}(F)$ defined in ( twisted sectors, finds instead the following compact expression for the anomaly:

$$
\begin{array}{r}
I_{F T}^{\mathrm{tw}}(F, R, G)=-i \sum_{l \in S_{l}} N_{l}\left[\operatorname{ch}_{(\mathbf{2 7}, \mathbf{1})}(F) \operatorname{ch}_{\theta_{i}^{l}-\frac{1}{3}}(F) \operatorname{ch}_{-\left(1-\theta_{i}^{l}\right)}(G)+\right. \\
\left.+\sum_{s} \operatorname{ch}_{q_{i}(s)}(F) \operatorname{ch}_{n_{i}(s)}(G)\right] \widehat{A}(R),
\end{array}
$$




\begin{tabular}{|c|c|c|c|c|c|}
\hline$P$ & $v$ & $n_{T}$ & $H$ & \multicolumn{2}{|c|}{ Matter } \\
\hline $\mathbb{Z}_{3}$ & $\left(\frac{1}{3}, \frac{1}{3}, \frac{-2}{3}\right)$ & 9 & $\overline{\mathrm{SU}(3)}$ & $1:(\mathbf{2 7}, \mathbf{3})_{-\delta_{i}^{1,2,3}}$ & $\begin{aligned} g: & (\mathbf{2 7}, \mathbf{1})_{-\rho_{i}^{1,2,3}} \\
& (\mathbf{1}, \overline{\mathbf{3}})_{-\left(\rho_{i}^{1}+\delta_{i}^{1,2,3}\right)}\end{aligned}$ \\
\hline $\mathbb{Z}_{7}$ & $\left(\frac{1}{7}, \frac{2}{7}, \frac{-3}{7}\right)$ & 3 & $\mathrm{U}(1)^{2}$ & $\begin{aligned} 1: & \left(\mathbf{2 7}_{0,2}\right)_{-\delta_{i}^{1}} \\
& \left(\mathbf{2 7}_{1,-1}\right)_{-\delta_{i}^{2}} \\
& \left(\mathbf{2 7}_{1,3}\right)_{-\delta_{i}^{3}} \\
& \left(\mathbf{1}_{1,-3}\right)_{-\delta_{i}^{1}} \\
& \left(\mathbf{1}_{-2,0}\right)_{-\delta_{i}^{2}} \\
& \left(\mathbf{1}_{-1,-1}\right)_{-\delta_{i}^{3}}\end{aligned}$ & $\begin{aligned} g: & \left(\mathbf{2 7}_{\frac{-2}{7}, \frac{-4}{7}}\right)_{-\rho_{i}^{1}} \\
& \left(\mathbf{1}_{\frac{-2}{7}}, \frac{-18}{7}\right)_{-\left(\rho_{i}^{1}+\delta_{i}^{1}\right)} \\
& \left(\mathbf{1}_{\frac{-9}{7}}, \frac{3}{7}\right)_{-\left(\rho_{i}^{1}+\delta_{i}^{2}\right)} \\
& \left(\mathbf{1}_{\frac{5}{7}, \frac{3}{7}}\right)_{-\left(\rho_{i}^{1}+\delta_{i}^{3}\right)} \\
& \left(\mathbf{1}_{\frac{-9}{7}}, \frac{3}{7}\right)_{-\left(\rho_{i}^{1}+2 \delta_{i}^{1}\right)} \\
& \left(\mathbf{1}_{\frac{5}{7}, \frac{3}{7}}\right)_{-\left(\rho_{i}^{1}+2 \delta_{i}^{2}\right)} \\
& \left(\mathbf{1}_{\frac{5}{7}, \frac{3}{7}}\right)_{-\left(\rho_{i}^{1}+\delta_{i}^{1}-\delta_{i}^{3}\right)} \\
& \left(\mathbf{1}_{\frac{5}{7}, \frac{3}{7}}\right)_{-\left(\rho_{i}^{1}+2 \delta_{i}^{1}+\delta_{i}^{2}\right)} \\
& \left(\mathbf{1}_{\frac{5}{7}, \frac{3}{7}}\right)_{-\left(\rho_{i}^{1}+4 \delta_{i}^{1}\right)}\end{aligned}$ \\
\hline & & & & $\begin{aligned} & g^{2}:\left(\mathbf{2 7}_{\frac{3}{7}, \frac{-1}{7}}\right)_{-\rho_{i}^{2}} \\
&\left(\mathbf{1}_{\frac{10}{7}, \frac{6}{7}}\right)_{-\left(\rho_{i}^{2}+\delta_{i}^{3}\right)} \\
&\left(\mathbf{1}_{\frac{3}{7}, \frac{-15}{7}}\right)_{-\left(\rho_{i}^{2}+\delta_{i}^{1}\right)} \\
&\left(\mathbf{1}_{\frac{-4}{7}, \frac{6}{7}}\right)_{-\left(\rho_{i}^{2}+\delta_{i}^{2}\right)} \\
&\left(\mathbf{1}_{\frac{3}{7}, \frac{-15}{7}}\right)_{-\left(\rho_{i}^{2}+2 \delta_{i}^{3}\right)} \\
&\left(\mathbf{1}_{\frac{-4}{7}, \frac{6}{7}}\right)_{-}\left(\rho_{i}^{2}+2 \delta_{i}^{1}\right) \\
&\left(\mathbf{1}_{\frac{-4}{7}, \frac{6}{7}}\right)_{-\left(\rho_{i}^{2}+\delta_{i}^{3}-\delta_{i}^{2}\right)} \\
&\left(\mathbf{1}_{\frac{-4}{7}, \frac{6}{7}}\right)_{-\left(\rho_{i}^{2}+2 \delta_{i}^{3}+\delta_{i}^{1}\right)} \\
&\left(\mathbf{1}_{\frac{-4}{7}, \frac{6}{7}}\right)_{-\left(\rho_{i}^{2}+4 \delta_{i}^{3}\right)} \\
&\end{aligned}$ & $\begin{aligned} & g^{4}:\left(\mathbf{2 7}_{\frac{-1}{7}, \frac{5}{7}}\right)_{-\rho_{i}^{4}} \\
&\left(\mathbf{1}_{\frac{-8}{7}}, \frac{12}{7}\right)_{-\left(\rho_{i}^{4}+\delta_{i}^{2}\right)} \\
&\left(\mathbf{1}_{\frac{6}{7}, \frac{22}{7}}\right)_{-\left(\rho_{i}^{4}+\delta_{i}^{3}\right)} \\
&\left(\mathbf{1}_{\frac{-1}{7}, \frac{-9}{7}}\right)_{-\left(\rho_{i}^{4}+\delta_{i}^{1}\right)} \\
&\left(\mathbf{1}_{\frac{6}{7}, \frac{21}{7}}\right)_{-\left(\rho_{i}^{4}+2 \delta_{i}^{2}\right)} \\
&\left(\mathbf{1}_{\frac{-1}{7}, \frac{-9}{7}}\right)_{-\left(\rho_{i}^{4}+2 \delta_{i}^{3}\right)} \\
&\left(\mathbf{1}_{\frac{-1}{7}, \frac{-9}{7}}\right)_{-\left(\rho_{i}^{4}+\delta_{i}^{2}-\delta_{1}^{i}\right)} \\
&\left(\mathbf{1}_{\frac{-1}{7}, \frac{-9}{7}}\right)_{-\left(\rho_{i}^{4}+2 \delta_{i}^{2}+\delta_{3}^{i}\right)} \\
&\left(\mathbf{1}_{\frac{-1}{7}, \frac{-9}{7}}\right)_{-\left(\rho_{i}^{4}+4 \delta_{i}^{2}\right)} \\
&\end{aligned}$ \\
\hline
\end{tabular}

Table 1: The $\mathbb{Z}_{3}$ and $\mathbb{Z}_{7}$ models, with their twist $v$, enhancement gauge group $H$, number $n_{T}$ of Kähler moduli and matter spectrum. The representations refer to the visible gauge group $G=E_{6} \times H$ and have multiplicity equal to the number of fixed points in each twisted sector. The modular weights $n_{i}$ of each matter field appear as a subindex $\left(\rho_{i}^{l}=1-\theta_{i}^{l}\right)$.

where the last sum runs over all the massless $E_{6}$-neutral twisted states $s$, with modular weights $n_{i}(s)$ and $\mathrm{SU}(3)$ charge vector $q_{i}(s)$. The structure of these states is described in more detail in appendix from the elliptic genus, and the correct modular weights and charge vectors are found.

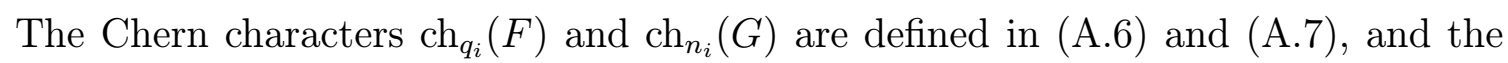
complete massless spectra are reported in table

As discussed in last section, consistency requires that the total anomaly must take a factorized form, as a consequence of modular invariance. We will now verifiy this explicitly for the two models under consideration. Irreducible gauge-anomalies cancel between untwisted an twisted sectors. Similarly, the structure of the cubic target-space anomalies drastically simplifies in the total results, in such a way to allow a simple factorization compatible with modular invariance. 
$\mathbb{Z}_{3}$ model. For the $\mathbb{Z}_{3}$ model, $H=\mathrm{SU}(3)$. Consider first the untwisted sector. The twisted Chern characters appearing in (3.2. $\left.\overline{8}_{1}\right)$ are easily written in terms of the ordinary ones. Defining $\alpha=\exp 2 \pi i / 3$, one finds: ${ }^{8}$

$$
\begin{aligned}
I_{F T}^{\text {untw. }}=\frac{1}{6} \sum_{k=1}^{2} C_{k}[ & \widehat{A}_{k}(G) \widehat{G}(R)+\widehat{G}_{k}(G) \widehat{A}(R)+ \\
& +\left(\operatorname{ch}_{\mathbf{2 4 8}}(F)+\operatorname{ch}_{(\mathbf{7 8}, \mathbf{1})}(F)+\operatorname{ch}_{(\mathbf{1}, \mathbf{8})}(F)+\right. \\
& \left.\left.+2 \alpha^{k} \operatorname{ch}_{(\mathbf{2 7}, \mathbf{3})}(F)\right) \widehat{A}_{k}(G) \widehat{A}(R)\right]
\end{aligned}
$$

Consider next the twisted sector. As can be seen from table ${ }_{1}^{1}$, there is a triplet of non-oscillator states, and from ( $\left({ }_{3}^{-} . \overline{2} \overline{9}_{1}^{\prime}\right)$ one gets:

$I_{F T}^{\mathrm{tw}}(F, R, G)=-27 i\left[\operatorname{ch}_{(\mathbf{2 7}, \mathbf{1})}(F) \operatorname{ch}_{-\left(1-\theta_{i}^{1}\right)}(G)+\sum_{j=1}^{3} \operatorname{ch}_{(\mathbf{1}, \overline{\mathbf{3}})}(F) \operatorname{ch}_{-\left(1-\theta_{i}^{1}+\delta_{i}^{j}\right)}(G)\right] \widehat{A}(R)$.

It is then straightforward to compute the explicit expression of the total anomaly. One finds as expected a factorized expression:

$$
I_{F T}=\frac{15}{2(2 \pi)^{3}}\left(\sum_{i=1}^{3} G_{i}\right)\left(\operatorname{tr} R^{2}-\operatorname{tr}_{E_{8}} F^{2}-\frac{1}{3} \operatorname{tr}_{E_{6}} F^{2}-2 \operatorname{tr}_{\mathrm{SU}(3)} F^{2}+2 \sum_{i=1}^{3} G_{i}^{2}\right) .
$$

We have used the standard definition $\operatorname{Tr}_{E_{8}} F^{2}=30 \operatorname{tr}_{E_{8}} F^{2}$ for the 248 of $E_{8}$ and the relation $\operatorname{Tr}_{E_{6}} F^{2}=4 \operatorname{tr}_{E_{6}} F^{2}$ between the $\mathbf{7 8}$ and 27 of $E_{6}$.

$\mathbb{Z}_{7}$ model. For the $\mathbb{Z}_{7}$ model, $H=\mathrm{U}(1) \times \mathrm{U}(1)$. The definition of the embedding of $\mathrm{U}(1) \times \mathrm{U}(1) \subset \mathrm{SU}(3)$ is completely arbitrary, and for convenience we will not yet make any precise choice. In fact, together with the $\mathrm{U}(1) \subset E_{6}$, there are three $\mathrm{U}(1)_{i}$, $i=1,2,3$, representing the unbroken part of the $\mathrm{SO}(6)$ group of internal rotations. There is then a natural choice for this subgroup, in which $\mathrm{U}(1)_{i}$ corresponds to rotations within the $i$-th internal torus $T_{i}^{2}$. The $i$-th component of the charge vector $q_{i}$, introduced above for a generic state contributing to the anomaly, then represents the charge with respect to this $\mathrm{U}(1)_{i}$. One can therefore keep the charge vector $q_{i}$ to describe the $H$ quantum numbers; since $q_{i}$ satisfies $\sum_{i} q_{i}=0$, it is always in $\mathrm{SU}(3)$ and never provides additional charge under $\mathrm{U}(1) \subset E_{6}$. We will adopt this notation below, as was also done in [3]

Consider first the untwisted sector. Again, the twisted Chern characters are easily reduced to the ordinary ones. In particular, one finds three replicas of matter contributions, which it will prove convenient to label with $l=1,2,4$ in the same

\footnotetext{
${ }^{8}$ We have that $\alpha^{k} \operatorname{ch}(F)$ and $\alpha^{-k} \operatorname{ch} h_{R}(-F)$ give the same contribution when summing over $k$.
} 
way as the three twisted sectors, and define the corresponding permutation degree $d_{l}$ such that $d_{1}=0, d_{2}=1, d_{4}=2$. Defining then $\alpha=\exp 2 \pi i / 7$, one finds:

$$
\begin{aligned}
I_{F T}^{\mathrm{untw}}=\frac{1}{14} \sum_{k=1}^{6} C_{k}[ & \widehat{A}_{k}(G) \widehat{G}(R)+\widehat{G}_{k}(G) \widehat{A}(R)+ \\
& +\left(\operatorname{ch}_{\mathbf{2 4 8}}(F)+\operatorname{ch}_{(\mathbf{7 8 , 1})}(F)+2+\right. \\
& \left.\left.+2 \sum_{l=1,2,4} \alpha^{l k}\left(\operatorname{ch}_{\mathbf{2 7}}{ }_{-\delta_{i}^{1+d_{l}}+\delta_{i}^{2+d_{l}}}(F)+\operatorname{ch}_{\delta_{i}^{d_{l}}}(F)\right)\right) \widehat{A}_{k}(G) \widehat{A}(R)\right] .
\end{aligned}
$$

Consider next the twisted sectors. The three sectors $l=1,2,4$ are related by permutations, and in each of them there are eight massless states, neutral under $E_{6}$, whose modular weights and charges (with a specific choice of $U(1)$ charges, see below) are reported in table ${ }_{-1}^{1}$. The form of the anomaly then is

$$
\begin{aligned}
& I_{F T}^{\mathrm{tw} .}(F, R, G)=-7 i \sum_{l=1,2,4}\left[\operatorname{ch}_{\mathbf{2 7}_{\theta_{i}-\frac{1}{3}}}(F) \operatorname{ch}_{-\left(1-\theta_{i}\right)}(G)+\right. \\
& +\sum_{j=1,2,3} \operatorname{ch}_{\mathbf{1}_{\theta_{i}-\delta_{i}^{j}}^{j-d_{l}}}(F) \mathrm{ch}_{-\left(1-\theta_{i}+\delta_{i}^{j-d_{l}}\right)}(G)+ \\
& +\sum_{h=1,2} \operatorname{ch}_{\mathbf{1}_{\theta_{i}-\delta_{i}}^{h-d_{l}+1}}(F) \mathrm{ch}_{-\left(1-\theta_{i}+2 \delta_{i}^{h-d_{l}}\right)}(G)+ \\
& +\operatorname{ch}_{\mathbf{1}_{i}-\delta_{i}^{3-d_{l}}}(F) \mathrm{ch}_{-\left(1-\theta_{i}+4 \delta_{i}^{3-d_{l}}\right)}(G)+ \\
& +\operatorname{ch}_{\mathbf{\theta}_{i}-\delta_{i}^{3-d_{l}}}(F) \mathrm{ch}_{-\left(1-\theta_{i}+2 \delta_{i}^{1-d_{l}}+\delta_{i}^{2-d_{l}}\right)}(G)+ \\
& \left.+\operatorname{ch}_{\mathbf{\theta}_{\theta_{i}-\delta_{i}^{3}}^{3-d_{l}}}(F) \operatorname{ch}_{-\left(1-\theta_{i}+\delta_{i}^{1-d_{l}}-\delta_{i}^{3-d_{l}}\right)}(G)\right] \widehat{A}(R) .
\end{aligned}
$$

In order to compute the explicit form of $\left(\overline{3}_{3} . \overline{3}\right.$ i $)$, one has to choose a specific embedding of the U(1)'s. One can define them through the corresponding charges $Q_{1,2}$ assigned to the generic $\mathrm{SU}(3)$ vector $q_{i}$. We take the combinations $Q_{1}(q)=\left(q_{2}-q_{3}\right) / 2 \sqrt{2}$ and $Q_{2}(q)=\left(2 q_{1}-q_{2}-q_{3}\right) / 2 \sqrt{6}$, which are canonically normalized. Notice that the (non-canonically normalized) charge $Q_{0}$ with respect to the $\mathrm{U}(1) \subset E_{6}$ is given by $Q_{0}(q)=2\left(q_{1}+q_{2}+q_{3}\right)$, which vanishes for any $q_{i}$ occurring here. The charges obtained in this way for each state are reported in table $i_{-i}$ in units of $1 / 2 \sqrt{2}$ for $Q_{1}$ and $1 / 2 \sqrt{6}$ for $Q_{2}$. After a straightforward but lengthy computation, one finds:

$$
I_{F T}=\frac{15}{2(2 \pi)^{3}}\left(\sum_{i=1}^{3} G_{i}\right)\left(\operatorname{tr} R^{2}-\operatorname{tr}_{E_{8}} F^{2}-\frac{1}{3} \operatorname{tr}_{E_{6}} F^{2}-\frac{1}{2} F_{1}^{2}-\frac{1}{2} F_{2}^{2}+2 \sum_{i=1}^{3} G_{i}^{2}\right) .
$$

Notice that all U(1) anomalies cancel. 


\subsection{GS terms}

The GS term can be computed from (2.2.7. has first of all to evaluate ( determined. One finds the following results for the two models under consideration:

$$
\begin{aligned}
& \mathbb{Z}_{3}: X_{4}=\operatorname{tr} R^{2}-\operatorname{tr}_{E_{8}} F^{2}-\frac{1}{3} \operatorname{tr}_{E_{6}} F^{2}-2 \operatorname{tr}_{\mathrm{SU}(3)} F^{2}+2 \sum_{i=1}^{3} G_{i}^{2}, \\
& \mathbb{Z}_{7}: X_{4}=\operatorname{tr} R^{2}-\operatorname{tr}_{E_{8}} F^{2}-\frac{1}{3} \operatorname{tr}_{E_{6}} F^{2}-\frac{1}{2} F_{1}^{2}-\frac{1}{2} F_{2}^{2}+2 \sum_{i=1}^{3} G_{i}^{2} .
\end{aligned}
$$

The gauge part of $\left({ }_{3} . \overline{3} \bar{b}_{i}\right)$ and $\left({ }_{3} . \overline{3} \overline{7}_{1}\right)$ is universal, i.e. independent of the gauge group factor, as required for a GS mechanism involving only the NSNS axion [i 19 . This can be easily verified by using the values $C\left(E_{8}\right)=30, C\left(E_{6}\right)=12$ and $C(\mathrm{SU}(3))=3$ for the quadratic Casimirs to evaluate gauge traces; one finds $\operatorname{tr}_{E_{8}} F^{2}=1 / 2 F_{E_{8}}{ }^{n} \wedge F_{E_{8} n}$, $1 / 3 \operatorname{tr}_{E_{6}} F^{2}=1 / 2 F_{E_{6}}{ }^{n} \wedge F_{E_{6 n}}, 2 \operatorname{tr}_{\mathrm{SU}(3)} F^{2}=1 / 2 F_{\mathrm{SU}(3)}{ }^{n} \wedge F_{\mathrm{SU}(3)}$, where the index $n$ is summed over all the generators of the particular group factor.

Notice that the explicit form of $X_{4}$ in both models requires a very special factorization of the one-loop anomalies ( anomalies have to vanish, as indeed happens in both models, but thanks to a very non-trivial compensation of the contributions of all fields.

\section{Low-energy interpretation}

We shall now analyse the results of the string theory computation of last section from a low-energy supergravity point of view. In particular, we will attempt to make contact with the results of [1]

A first important issue is the form of the anomalies derived within string theory,

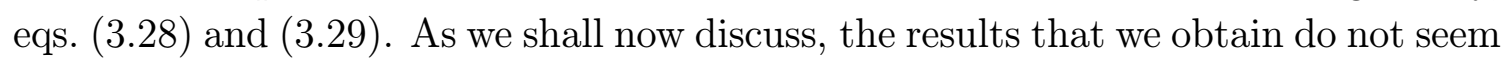
to match in all details the expectations based on a low-energy supergravity approach. A number of important qualitative differences can be understood and recognized to correspond to the different frames used in the two contexts: the Einstein and the string frames. The dependence on the gauge and gravitational curvatures is encoded in the standard characteristic classes, as expected. As for the dependence on the target-space curvature, on the other hand, there is an important qualitative difference between the contributions of untwisted and twisted states; for the former, this dependence resembles a gravitational dependence, whereas for the latter it is more similar to a gauge dependence. This suggest that twisted states feel the target-space background only through the target-space connection $Z_{\mu}=-i \sum_{i}\left(T_{i}+\bar{T}_{\bar{\imath}}\right)^{-1} \partial_{\mu}\left(T_{i}-\bar{T}_{\bar{\imath}}\right)$, whereas untwisted states have some additional sensitivity to it. In fact, this is recognized to be nothing but the effect of the moduli-dependent compactification volume 
$V=\prod_{i}\left(T_{i}+\bar{T}_{\bar{\imath}}\right)$, arising from the determinant of the internal metric. In the string frame, such a volume factor arises by dimensional reduction in the untwisted sector, but is clearly absent in twisted sectors. From a purely low-energy supergravity point of view, in turn, it is possible and in fact convenient to move to the so-called Einstein frame, where in particular the volume dependence in the untwisted sector part of the effective action is reabsorbed through a suitable definition of the metric. In this framework, it is then plausible to expect that target-space anomalies can be computed as simple gauge anomalies. This is indeed the approach adopted in [21]. Clearly, the final result for the total anomaly should be identical in the two approaches, and at most, one expects a reshuffling of the single contributions to the anomaly from those states affected by the frame redefinition. In fact, the comparison between the string-derived anomaly encoded in $\left({ }^{2} \overline{3} . \overline{2} \overline{8}_{1}^{\prime}\right)-\left({ }^{\prime} \overline{3} . \overline{2} \overline{2}_{1}^{\prime}\right)$ and that expected from an Einstein frame supergravity approach as in [21] can be done, and seems to lead to a discrepancy.

Consider first mixed target-space-gauge and target-space-gravitational anomalies. The relevant components of the polynomial $I_{F T}$ given by the sum of $(28)$ and ( $\left(13.2 \overline{9}_{1}^{\prime}\right)$ are found to be of the form $I_{G F F}=1 / 2(2 \pi)^{3} \sum_{i, a} b_{a}^{i} G_{i} \wedge F_{a}{ }^{n} \wedge F_{a n}$ and $I_{G R R}=1 / 48(2 \pi)^{3} \sum_{i} b^{i} G_{i} \wedge R^{\mu \nu} \wedge R_{\mu \nu}$, with coefficients $b_{a}^{i}$ and $b_{i}$ given by:

$$
\begin{aligned}
b_{a}^{i} & =-C\left(G_{a}\right)+\sum_{R_{a}} T\left(R_{a}\right)\left(1+2 n_{R_{a}}^{i}\right), \\
b^{i} & =21+1+\delta_{T}-\operatorname{dim}(G)+\sum_{\alpha}\left(1+2 n_{\alpha}^{i}\right) .
\end{aligned}
$$

The correct modular weight $n_{i}$ is automatically obtained for each state. In $b_{a}^{i}$, the first contribution is from the gauge bosons and involves the quadratic Casimir $C\left(G_{a}\right)$ of the relevant group factor $G_{a}$, whereas the sum in the second term runs over charged states with non-trivial representations $R_{a}$ with respect to the gauge group factor $G_{a}$, with character $T\left(G_{a}\right)$. In $b^{i}$ instead, the 21 corresponds to the gravitino, the 1 to the dilatino, the quantity $\delta_{T}$ to the untwisted moduli, and the sum in the last term is over all matter states $\alpha$. The above expressions for the coefficients $b_{a}^{i}$ and $b_{i}$ coincide term by term with the well-known results of $\left[\begin{array}{l}1 \\ 1\end{array} \bar{g}_{1}, i_{1}^{1} \overline{2} 0\right]$, for each single contribution, showing perfect agreement for mixed anomalies

In the case of pure target-space anomalies, instead, the string-derived results extracted from ( $\left(5.28_{1}^{\prime}\right)-\left(\sqrt[5]{5} .29_{1}^{\prime}\right)$ and those expected from the supergravity analysis of [2] differ quite radically in the untwisted sector, although agreement is found for twisted sectors. Not only the single contributions from each untwisted state disagree, but they also sum up to different total results, thus showing a true discrepancy.

The anomaly we find therefore differs concretely from the one expected from previous analyses in the literature, beyond the terms linear in target-space curvatures. The difference could be related to subtleties associated to the compositeness of the target-space connection, which could invalidate the analogy with a gauge connection 
when many of them occur as external states. As a matter of fact, it turns out that the total anomaly derived from our string computation nicely factorizes, whereas we have checked that the total anomaly computed by assuming that the target-space dependence is analogous to the gauge dependence does not factorize. We take this as an indirect argument in favour of our results, and therefore assume that they should be reproduced by a more careful supergravity analysis.

The two explicit examples that we have analysed in detail exhibit a certain number of properties that we expect to be quite general, and valid for any orbifold model free of $U(1)$ anomalies and fixed planes. Defining the quantity $\operatorname{tr} F^{2}$ to represent the appropriate sum of terms over the various group factors $G_{a}$ arising in a given model: $\operatorname{tr} F^{2}=1 / 2 \sum_{a} F_{a}{ }^{n} \wedge F_{a n}$, the anomaly can be written in a universal way as $I_{F T}=X_{2} \wedge X_{4}$ with:

$$
\begin{aligned}
& X_{2}=\frac{15}{2(2 \pi)^{3}}\left(\sum_{i=1}^{3} G_{i}\right) \\
& X_{4}=\operatorname{tr} R^{2}-\operatorname{tr} F^{2}+2 \sum_{i=1}^{3} G_{i}^{2} .
\end{aligned}
$$

These same quantities enter also the GS term $-2 \pi B \wedge X_{2}$ and the modified Bianchi identity $d H=X_{4}$. These general results encode full information about all possible target-space, gauge and gravitational anomalies in four-dimensional heterotic vacua without anomalous U(1)'s and threshold corrections, as well as the GS mechanism through which they are cancelled. The pure target-space part is a novel result, at both the field-theory and the string-theory level. The generalization to models with an anomalous $\mathrm{U}(1)$ does not present any technical problem, and only the twoform $X_{2}$ entering the GS term is expected to be modified, through additional terms proportional to the field strength of the anomalous $\mathrm{U}(1)$. Models with fixed planes and threshold corrections, instead, do present interesting new features [i] $\left.{ }_{1}^{1} \overline{\bar{T}_{1}}\right]$.

The details of the GS mechanism of anomaly cancellation occurring at the level of low-energy effective action do not present any qualitative novelty. As usual, the cancellation mechanism can be understood both in a linear and a chiral multiplet

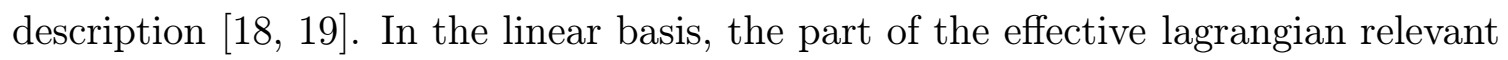
to anomaly cancellation is given by

$$
L_{l}=-\frac{1}{12}\left|d B-X_{4}^{(0)}\right|^{2}-2 \pi B \wedge X_{2} .
$$

The modified kinetic term of $B$ requires that $\delta B=X_{4}^{(1)}$ under gauge, gravitational or target-space transformations. The GS term induces an anomaly $\delta L_{l}=-2 \pi X_{2} \wedge X_{4}^{(1)}$, which is equivalent to the $\mathrm{WZ}$ descent of $I_{G S}=-X_{2} \wedge X_{4}$, modulo irrelevant local terms. This exactly cancels the field-theory anomaly $I_{F T}=X_{2} \wedge X_{4}$. In the chiral 
basis, the relevant lagrangian for the axion field $\chi$ is instead:

$$
L_{c}=\frac{1}{2}\left|d \chi-X_{2}^{(0)}\right|^{2}-2 \pi \chi X_{4} .
$$

The shift in the kinetic term now involves only the target-space connection. This requires $\delta \chi=X_{2}^{(1)}$ under target-space transformations. The GS term induces an anomaly $\delta L_{c}=-2 \pi X_{2}^{(1)} \wedge X_{4}$, which is again equivalent to the WZ descent of $I_{G S}=-X_{2} \wedge X_{4}$, cancelling as before the field-theory anomaly $I_{F T}=X_{2} \wedge X_{4}$. In both approaches, one can easily generalize the above results for the CP-odd part to full superspace expressions, encoding for instance also CP-even target-space anomalies.

\section{Geometric structure and relation to torsion}

The general structure of the anomalies derived in the previous sections can be understood quite nicely from a geometrical point of view, and reveals some interesting

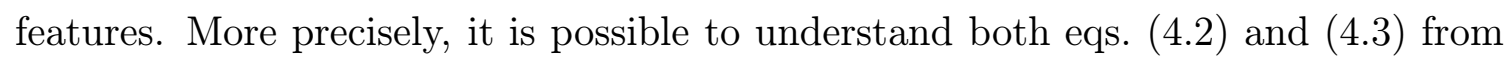
a ten-dimensional point of view.

Let us begin by recalling some well-known facts about the ten-dimensional case. The tree-level effective lagrangian of the $D=10$ heterotic theory exhibits a modified kinetic term for the NSNS two-form $B$, involving the field-strength $H=d B-Y_{4}^{(0)}$, where the Chern-Simons three-form $Y_{4}^{(0)}$ is defined as the WZ descent of the fourform

$$
Y_{4}=\operatorname{tr} R^{2}-\operatorname{tr} F^{2}
$$

Correspondingly, the NSNS field has a modified Bianchi identity $d H=Y_{4}$ and transforms non-trivially as $\delta B=Y_{4}^{(1)}$ under gauge transformations or diffeomorphisms. The well-known GS mechanism then occurs through the variation of the stringgenerated one-loop effective coupling $-2 \pi B \wedge Y_{8}$, where the eight-form $Y_{8}$ is given by

$$
Y_{8}=\frac{1}{8} \operatorname{tr} R^{4}+\frac{1}{32}\left(\operatorname{tr} R^{2}\right)^{2}+\frac{1}{4}\left(\operatorname{tr} F^{2}\right)^{2}-\frac{1}{8} \operatorname{tr} F^{2} \operatorname{tr} R^{2} .
$$

An important thing to note is that the two-form $R$ entering both ( fact a generalized curvature hiding also a dependence on the torsion $H .{ }^{9}$ Indeed, it has been shown in [3] and world-sheet conformal invariance, fix unambiguously the connection $\omega$ which defines $R$ in the above equations to be the sum of the usual spin connection and the torsion connection constructed from $H$. This is also clear from a direct computation of the anomaly along the lines of section effect of torsion through vertex operators for the $B$ field.

Let us now consider an orbifold compactification leading to $D=4$. The spectrum of the states arising from the untwisted spectrum can easily be deduced starting from

\footnotetext{
${ }^{9}$ The Bianchi identity for $H$ then must be understood and solved iteratively, in an $\alpha^{\prime}$ expansion.
} 
$D=10$. In particular, the $D=10$ graviton and NSNS two-form $B$ give rise (in addition to the $D=4$ graviton, two-form $B$ and possibly other scalars) to the three diagonal untwisted complex fields (13.3). In standard orbifold compactifications, the internal metric and NSNS flux are fixed, and the vacuum expectation value of the $T$-moduli is constant, implying in particular that there is no background torsion in the internal manifold. However, a non-trivial generalized connection is induced by the fluctuations of the complexified internal metric represented by the untwisted $T$-moduli. One finds

$$
\omega_{\mu}^{i \bar{\imath}}=Z_{\mu i}
$$

where $Z_{i}$ are precisely the target-space connections $\left({ }_{2}^{5} .1 \overline{6}_{1}^{\prime}\right)$. Correspondingly, the internal components of the generalized curvature two-form $R$ do not vanish, but rather $R_{i \bar{\imath}}=G_{i}$, and the ten-dimensional quantity $\operatorname{tr} R^{2}$ decomposes into its fourdimensional analogue, plus a contribution coming from the internal components:

$$
\operatorname{tr}_{D=10} R^{2}=\operatorname{tr}_{D=4} R^{2}+2 \sum_{i=1}^{3} G_{i}^{2} .
$$

The general structure ( mensional reduction of the well-known expression (1, $\left.\overline{1}_{1}^{\prime}\right)$ for $Y_{4}$ in $D=10$. A similar

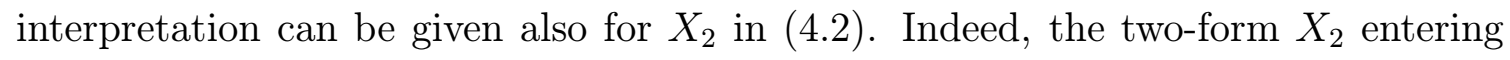
the GS term in a $D=4$ model can be obtained by integrating the $D=10$ GS term $Y_{8}$ given by (15.2) over the compactification manifold. Much in the same way as $\mathrm{U}(1)$ terms in $X_{2}$ comes from couplings in (15.2.

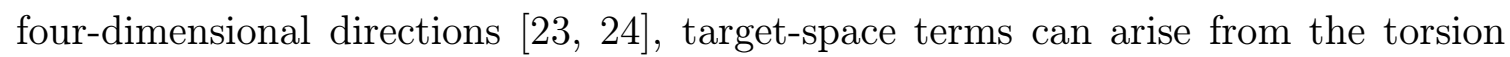

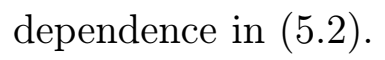

Using the geometric interpretation just described, one can also obtain a much better understanding of the various characteristic classes appearing in the contri-

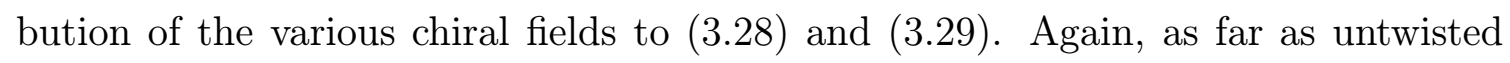
states are concerned, it is convenient and justified to reinterpret the result from a

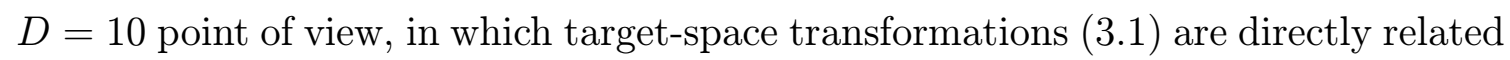
to internal reparametrizations of the orbifold. The first line in ( from a chiral gravitino in $D=10$, which gives rise, when dimensionally reduced to $D=4$, to a chiral gravitino transforming as an internal spinor (first term), plus chiral spinors transforming as an internal gravitino (second term), with respect to the reparametrizations of the orbifold induced by ( in $\left(\overline{3} . \overline{2} g^{\prime}\right)$, instead, come from charged $D=10$ chiral spinors, the gauginos, which give rise to multiplets of chiral spinors in $D=4$ transforming as internal spinors. Twisted states arise from given fixed points of the orbifold and therefore cannot be understood from a $D=10$ point of view. It is clear from the form of $\left(\overline{3} . \overline{2} 9^{\prime}\right)$ that they are simply $D=4$ spinors and transform just as $\mathrm{U}(1)$ charged fields under reparametrizations of the orbifold, with charges given by the modular weights $n_{i}$. 
As already noticed in section ${ }_{-1}^{1}$, the reason for the different structure of anomalies in untwisted and twisted sectors is related to the very different way in which the corresponding states arise geometrically.

\section{Conclusions}

We have shown that target-space anomalies in heterotic orbifold models can be understood on the same footing as gauge and gravitational anomalies, allowing for a more general and unified analysis of them in such models. All anomalies cancel through a GS mechanism mediated by the dilaton multiplet, thanks to a new term in the Bianchi identity for the NSNS $B$-field that involves the target-space curvature. The key property behind this cancellation is again modular invariance, and both the quantum anomaly and the classical GS term are encoded in a generalized elliptic genus with well-defined modular properties. Although we have focused on the simplest four-dimensional orbifold models, it is clear that the validity of these results extends straightforwardly to a much wider class of models, involving for example non-standard embedding, Wilson lines, or a different number of space-time dimensions. A similar analysis should also be possible for models with fixed planes and threshold corrections in $N=2$ sectors.

Several interesting novelties have emerged in the low-energy understanding of target-space symmetry as well. For instance, a precise relation to torsion has emerged, which leads to a nice geometric interpretation. Our results for quantum anomalies involving more than one target-space connection disagree from the naive field theory expectation based on the analogy with a gauge connection, suggesting interesting technical subtleties to be unravelled.

A very interesting application of target-space symmetries has recently emerged in the context of a possible duality between certain heterotic orbifold and type-

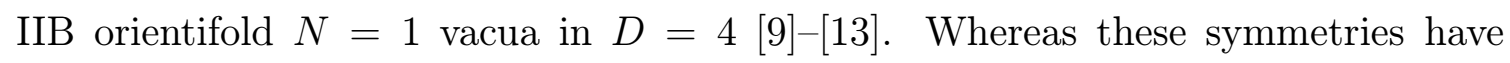
a good reason to persist quantum mechanically on the heterotic side (the underlying T-duality), they are apparently accidental on the orientifold side, and therefore constitute a very stringent test of the proposed duality. It has been shown recently in [i] that one-loop anomalies in the simplest $\mathbb{Z}_{3}$ and $\mathbb{Z}_{7}$ orientifold models are cancelled through a generalized GS mechanism mediated by several twisted RR fields, as proposed in [3] erotic models and those derived in [i] for type-IIB orientifold models demonstrate that at least the simplest vacua in both theories do indeed have the same targetspace symmetry and are free of any anomaly. In spite of other problems pointed out so far in the literature [4. 6 , this is quite a suggestive result in favour of the conjectured duality. Finally, it is interesting to note that the same peculiar structure of the target-space anomalies appears in both heterotic orbifolds and type-IIB orientifolds. 


\section{Acknowledgments}

It is a pleasure to thank W. Lerche and A. Uranga for very interesting discussions, and G. L. Cardoso for useful comments. C.A.S. also thanks the International School for Advanced Studies (ISAS-SISSA) for hospitality during the completion of this work.

\section{A. Characteristic classes}

The relevant characteristic classes appearing in the anomaly from chiral spinors and Rarita-Schwinger fields are the Roof-genus and the $G$-polynomial [3]7], functions of the gravitational curvature $R$ and defined in terms of the skew eigenvalues $\lambda_{a}$ of $R / 2 \pi$ as:

$$
\begin{aligned}
\widehat{A}(R) & =\prod_{a=1}^{D / 2} \frac{\lambda_{a} / 2}{\sinh \lambda_{a} / 2} \\
\widehat{G}(R) & =\prod_{a=1}^{D / 2} \frac{\lambda_{a} / 2}{\sinh \lambda_{a} / 2}\left(2 \sum_{b=1}^{D / 2} \cosh \lambda_{b}-1\right) .
\end{aligned}
$$

For the target-space dependence, similar characteristic classes turn out to appear; these are functions of the composite curvature $G=d Z$ and defined, in terms of $g_{i}=G_{i} / 2 \pi$, as

$$
\begin{aligned}
\widehat{A}_{k}(G) & =\prod_{i=1}^{3} \frac{\sin \left(\pi k v_{i}\right)}{\sin \left(g_{i} / 2+\pi k v_{i}\right)} \\
\widehat{G}_{k}(G) & =\prod_{i=1}^{3} \frac{\sin \left(\pi k v_{i}\right)}{\sin \left(g_{i} / 2+\pi k v_{i}\right)}\left(2 \sum_{j=1}^{3} \cos \left(g_{j}+2 \pi k v_{j}\right)-1\right) .
\end{aligned}
$$

The gauge dependence is influenced by the orbifold projection, which acts on the $\mathrm{SU}(3)$ part of the maximal gauge group and projects it to a subgroup $H$. The element $g^{k}$ of the orbifold group $\mathbb{Z}_{N}$ acts as $F / 2 \pi \rightarrow F / 2 \pi+2 \pi i k V$ on the rescaled gauge curvature of the visible gauge group, where $V$ is an $\mathrm{SU}(3)$ shift with skeweigenvalues $v_{i}$. This shift acts as $y_{i} \rightarrow y_{i}+2 \pi k v_{i}$ for $i=1,2,3$. It is then natural to define the following twisted Chern characters:

$$
\operatorname{ch}^{k}(F)=\operatorname{ch}\left(F+(2 \pi)^{2} k V\right) .
$$

Finally, it is convenient to define:

$$
\begin{aligned}
& \operatorname{ch}_{q_{i}}^{k}(F)=\exp \left\{i \sum_{i=1}^{3} q_{i}\left(y_{i}+2 \pi k v_{i}\right)\right\}, \\
& \operatorname{ch}_{n_{i}}^{k}(G)=\exp \left\{-i \sum_{i=1}^{3}\left(1+2 n_{i}\right)\left(\frac{g_{i}}{2}+\pi k v_{i}\right)\right\} .
\end{aligned}
$$




\section{B. Decomposition of characters}

We report in this appendix some useful explicit expressions for the Chern characters relevant to orbifold models with standard embedding. Recall first that the $\mathrm{SO}(16)$ group underlying $E_{8}$ decomposes naturally into $\mathrm{SO}(6) \times \mathrm{SO}(10)$, and the eight skew eigenvalues of the rescaled $\mathrm{SO}(16)$ curvature $F / 2 \pi\left(y_{i}, i=1, \ldots, 8\right)$ split into the three skew eigenvalues of the $\mathrm{SO}(6)$ curvature $\left(y_{i}, i=1, \ldots, 3\right)$ and the five skew eigenvalues of the $\mathrm{SO}(10)$ curvature $\left(y_{i}, i=4, \ldots, 8\right)$. The internal $\mathrm{SO}(6) \sim \mathrm{SU}(4)$ group further decomposes into $\mathrm{U}(1) \times \mathrm{SU}(3)$, the rescaled $\mathrm{U}(1)$ curvature being $\sum_{i} y_{i} / 6$. For standard embeddding, one of the two $E_{8}$ groups of the $D=10$ theory is broken to $E_{6} \times H$ in $D=4$, where the $E_{6}$ group is constructed from $\mathrm{SO}(10) \times \mathrm{U}(1)$ and $H$ is a subgroup of $\mathrm{SU}(3)$. The Chern characters of the relevant $E_{8}$ and $E_{6}$ representations can then be derived using their decomposition with respect to the defining $\mathrm{SO}(16)$ and $\mathrm{SO}(10)$ subgroups respectively.

The basic fundamental and positive/negative spinor representations of $\mathrm{SO}(2 n)$ are easily computed. One finds:

$$
\begin{aligned}
\operatorname{ch}_{\mathbf{2 n}}(F) & =2 \sum_{i=1}^{n} \cos y_{i} \\
\operatorname{ch}_{\mathbf{2}^{\mathbf{n}-\mathbf{1}}}(F) & =2^{n-1}\left(\prod_{i=1}^{n} \cos \frac{y_{i}}{2} \pm \prod_{i=1}^{n} i \sin \frac{y^{i}}{2}\right),
\end{aligned}
$$

in terms of the skew-eigenvalues $y_{i}$ of the field strength $F / 2 \pi$. The Chern character in the adjoint representation is then obtained as

$$
\operatorname{ch}_{\mathbf{n}(\mathbf{2 n}-\mathbf{1})}(F)=\frac{1}{2}\left[\operatorname{ch}_{\mathbf{2 n}}^{2}(F)-\operatorname{ch}_{\mathbf{2 n}}(2 F)\right] .
$$

The adjoint representation 248 of $E_{8}$ decomposes as $248=\mathbf{1 2 0} \oplus \mathbf{1 2 8}$ under $\mathrm{SO}(16)$. Its Chern character is then found to be

$$
\operatorname{ch}_{\mathbf{2 4 8}}(F)=8+128\left(\prod_{i=1}^{8} \cos \frac{y_{i}}{2}+\prod_{i=1}^{8} \sin \frac{y_{i}}{2}\right)+2 \sum_{i \neq j=1}^{8} \cos y_{i} \cos y_{j} .
$$

The relevant representations of $E_{6}$ and $\mathrm{SU}(3)$ are those appearing in the decomposition of the adjoint of $E_{8}$ under the maximal subgroup $E_{6} \times \mathrm{SU}(3)$ :

$$
\mathbf{2 4 8} \rightarrow(\mathbf{7 8}, \mathbf{1}) \oplus(\mathbf{1}, \mathbf{8}) \oplus(\mathbf{2 7}, \mathbf{3}) \oplus(\overline{\mathbf{2 7}}, \overline{\mathbf{3}}) .
$$

The adjoint $\mathbf{7 8}$ and "fundamental" $\mathbf{2 7}$ representations of $E_{6}$ further decompose as follows with respect to the $\mathrm{SO}(10) \times \mathrm{U}(1)$ :

$$
\begin{aligned}
& \mathbf{7 8} \rightarrow \mathbf{4 5}_{0} \oplus \mathbf{1 6} \mathbf{6}_{3} \oplus \overline{\mathbf{1 6}}_{-3} \oplus \mathbf{1}_{0}, \\
& \mathbf{2 7} \rightarrow \mathbf{1 6}_{-1} \oplus \mathbf{1 0}_{2} \oplus \mathbf{1}_{-4} .
\end{aligned}
$$


The $\mathrm{U}(1) \times \mathrm{SU}(3)$ part of the Chern characters is easily obtained by returning to the original $\mathrm{SO}(6)$ representations. For example, using the decomposition of the $\mathbf{4}$ of $\mathrm{SO}(6)$ as $\mathbf{1}_{\mathbf{3}} \oplus \mathbf{3}_{-\mathbf{1}}$ under $\mathrm{U}(1) \times \mathrm{SU}(3)$ and applying $\left(\overline{\mathrm{B}} . \overline{\mathbf{1}_{1}}\right)$, one can obtain the Chern characters in the $\mathbf{1}_{\mathbf{3}}$ and the $\mathbf{3}_{-\mathbf{1}}$ representations. Generalizing to an arbitrary $\mathrm{U}(1)$ charge, one finds

$$
\begin{aligned}
& \operatorname{ch}_{\mathbf{1}_{\mathbf{q}}}(F)=\exp \left(i q \sum_{i=1}^{3} \frac{y_{i}}{6}\right) \\
& \operatorname{ch}_{\mathbf{3}_{\mathbf{q}}}(F)=\exp \left(i(q-2) \sum_{i=1}^{3} \frac{y_{i}}{6}\right) \sum_{i=1}^{3} \exp i y_{i} .
\end{aligned}
$$

Similarly, the Chern character of the adjoint representation $\mathbf{8}$ of $\mathrm{SU}(3)$ is easily deduced from the decomposition $\mathbf{3} \otimes \overline{\mathbf{3}}=\mathbf{8} \oplus \mathbf{1}$. The explicit form of the Chern characters for the remaining relevant representations is then found to be:

$$
\begin{aligned}
\operatorname{ch}_{(\mathbf{7 8 , 1})}(F)= & +32\left[\cos \left(\sum_{i=1}^{3} \frac{y_{i}}{2}\right) \prod_{j=4}^{8} \cos \frac{y_{j}}{2}+\sin \left(\sum_{i=1}^{3} \frac{y_{i}}{2}\right) \prod_{j=4}^{8} \sin \frac{y_{j}}{2}\right]+ \\
& +2 \sum_{j \neq k=4}^{8} \cos y_{j} \cos y_{k}, \\
\operatorname{ch}_{(\mathbf{1}, \mathbf{8})}(F)= & 2+\sum_{i \neq j=1}^{3} \cos \left(y_{i}-y_{j}\right), \\
\operatorname{ch}_{(\mathbf{2 7 , 3})}(F)= & {\left[\exp \left(-i \sum_{i=1}^{3} y_{i}\right)+16 \exp \left(-i \sum_{i=1}^{3} \frac{y_{i}}{2}\right)\left(\prod_{j=4}^{8} \cos \frac{y_{j}}{2}+\prod_{j=4}^{8} \sin \frac{y_{j}}{2}\right)+\right.} \\
& \left.+2 \sum_{j=4}^{8} \cos y_{j}\right] \sum_{i=1}^{3} \exp i y_{i} .
\end{aligned}
$$

Notice finally that the decomposition $\left({ }^{-} \overline{\mathrm{B}}-\overline{4}\right)$ can be technically understood on the

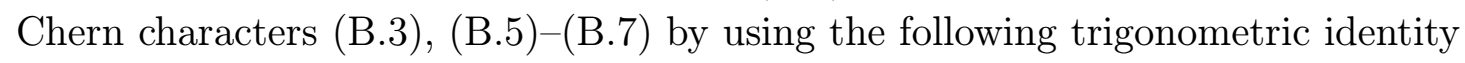

$$
\begin{aligned}
& \prod_{i=1}^{3} 2 \sin \frac{y_{i}}{2}=\sum_{i=1}^{3} 2 \sin \left(\sum_{j=1}^{3} \frac{y_{j}}{2}-y_{i}\right)-2 \sin \left(\sum_{i=1}^{3} \frac{y_{i}}{2}\right), \\
& \prod_{i=1}^{3} 2 \cos \frac{y_{i}}{2}=\sum_{i=1}^{3} 2 \cos \left(\sum_{j=1}^{3} \frac{y_{j}}{2}-y_{i}\right)+2 \cos \left(\sum_{i=1}^{3} \frac{y_{i}}{2}\right),
\end{aligned}
$$

implementing the $\mathrm{SO}(6) \rightarrow \mathrm{U}(1) \times \mathrm{SU}(3)$ decomposition.

\section{Limits of the partition functions}

In this appendix, we report some useful details about the computation of the $\tau \rightarrow i \infty$ limit of the partition functions $Z_{S T}(R), Z_{C}^{k, l}(G)$ and $Z_{\Gamma}^{k, l}(F)$ in eqs. ( 
In the untwisted sector, the limits are easily obtained, and one finds:

$$
\begin{aligned}
Z_{S T}(R) \rightarrow q^{-1 / 12}[\widehat{A}(R)+(\widehat{G}(R)-\widehat{A}(R)) q] \\
Z_{C}^{k, l=0}(G) \rightarrow \epsilon_{k} N_{k}^{-1 / 2} q^{-1 / 4}\left[1+\left(\widehat{A}_{k}(G)+\widehat{G}_{k}(G)\right) q\right] \\
Z_{\Gamma}^{k, l=0}(F) \rightarrow q^{-2 / 3}\left[1+\left(\operatorname{ch}_{\mathbf{2 4 8}}\left(F^{\prime}\right)+\operatorname{ch}_{(\mathbf{7 8 , 1})}(F)+\operatorname{ch}_{(\mathbf{1}, \mathbf{8})}^{k}(F)+\right.\right. \\
\left.\left.+\operatorname{ch}_{(\mathbf{2 7}, \mathbf{3})}^{k}(F)+\operatorname{ch}_{(\overline{\mathbf{2 7}, \overline{\mathbf{3}})}}^{k}(F)\right) q\right]
\end{aligned}
$$

Combining these expressions, the result ( $\left(\overline{3} . \overline{2} \overline{2} \overline{8}^{\prime}\right)$ is obtained.

In the twisted sector, the situation is more involved. Indeed, there are in general matter states with both vanishing and non-vanishing oscillator number $N_{L}$; correspondingly, there will be a complicated interference of terms between $Z_{C}$ and $Z_{\Gamma}$ in the limit $\tau \rightarrow i \infty$. For this reason, it is convenient to recall how these states arise in general. The mass-shell condition for a generic massless state in the $l$-th twisted sector reads

$$
N_{L}-1+\frac{\eta}{2}+\frac{(p+v l)^{2}}{2}=0
$$

where $\eta=\sum_{i=1}^{3} \theta_{i}^{l}\left(1-\theta_{i}^{l}\right)$ represents the shift in the zero-point energy in each model and takes the value $2 / 3$ for $\mathbb{Z}_{3}$ and $4 / 7$ for $\mathbb{Z}_{7}$. Such a state is in general created from the Fock vacuum, with certain numbers $m_{i}$ and $\tilde{m}_{i}$ of left- and right-moving bosonic creation operators in the $i$-th internal direction, determining an oscillator number $N_{L}$; it is associated with lattice vectors $p$ satisfying $(p+v)^{2}=2-2 N_{L}-\eta$. Since this is the most general massless twisted state, the relevant terms to keep in $Z_{S T}, Z_{C}$ and $Z_{\Gamma}$ are those of the type $Z_{S T} \sim \bar{q}^{-\frac{1}{12}}, Z_{C} \sim \bar{q}^{-\frac{1}{4}+\frac{\eta}{2}+N_{L}}$ and $Z_{\Gamma} \sim \bar{q}^{-\frac{2}{3}+\frac{(p+v)^{2}}{2}}$. Indeed, the corresponding term in the total partition function is then $Z \sim q^{N_{L}-1+\frac{\eta}{2}+\frac{(p+v)^{2}}{2}}$, and represents a massless contribution whenever the mass-shell condition ('C. satisfied. One can therefore restrict from the beginning to these types of terms when evaluating the partition functions for $\tau \rightarrow i \infty$.

The limit of the internal partition function $Z_{C}$ is easily obtained by using the infinite sum representation of $\theta$-functions. The integer $n$ labelling the infinite sum in the definition of the $\theta$-function associated to a given complex direction is related to the level of the bosonic oscillator that creates the corresponding state, and positive/negative values of $n$ correspond to left-/right- moving oscillators. Since all the massless states occurring in the considered models involve at most oscillators of lowest level, it is enough to keep the first two subleading terms (in $q$ ) beside the leading term, in each $\theta$-function. One then finds:

$$
Z_{C}^{k, l \neq 0}(G) \rightarrow-i \epsilon_{l}\left[\bar{q}^{-\frac{1}{4}+\frac{\eta}{2}} \operatorname{ch}_{-\left(1-\theta_{i}^{l}\right)}^{k}(G)+\sum_{s} \bar{q}^{-\frac{1}{4}+\frac{\eta}{2}+N_{L}(s)} \operatorname{ch}_{n_{i}(s)}^{k}(G)\right]
$$


with the following expressions for the oscillator number $N_{L}$ and the modular weight $n_{i}$ for the generic massless twisted state $s$ :

$$
\begin{aligned}
N_{L}(s) & =-\frac{1}{2} \sum_{i=1}^{3}\left[\left(m_{i}-\tilde{m}_{i}\right)\left(1-2 \theta_{i}^{l}\right)-\left(m_{i}+\tilde{m}_{i}\right)\right], \\
n_{i}(s) & =-\left(1-\theta_{i}^{l}+m_{i}-\tilde{m}_{i}\right) .
\end{aligned}
$$

Notice that the known expression [20̄0] for the modular weight of a generic oscillator state is recovered.

For the lattice partition function, one has to understand which kind of lattice vectors $p$ can occur for the generic state discussed above, and which representation is defined by the corresponding $q=p+v$. For the $N_{L}=0$ states, the allowed $p$ 's give rise to a $\mathbf{2 7}$ of $E_{6}$ and fixed charges in the $\mathrm{SU}(3)$ part, for both the $\mathbb{Z}_{3}$ and $\mathbb{Z}_{7}$ models. For the states with $N_{L} \neq 0$, the allowed $p$ 's lead to three distinct $q$ 's in the $\mathrm{SU}(3)$ part, which we shall label with $q^{a}, a=1,2,3$, giving rise to a triplet for $\mathbb{Z}_{3}$ and three groups of eight singlets for $\mathbb{Z}_{7}$. More precisely, the relevant part of the lattice partition function is found to be:

$$
Z_{\Gamma}^{k, l \neq 0}(F) \rightarrow \bar{q}^{\frac{1}{3}-\frac{\eta}{2}} \operatorname{ch}_{(\mathbf{2 7 , 1})}(F) \operatorname{ch}_{\theta_{i}^{l}+\frac{\epsilon_{l}-3}{6}}^{k}(F)+\sum_{a=1}^{3} \bar{q}^{-\frac{2}{3}+\left(q^{a}\right)^{2}} \operatorname{ch}_{\left(q^{a}\right)_{i}}^{k}(F) .
$$

One can check that the allowed $q(s)$ 's for an oscillator state $s$, among the three $q^{a}$, $a=1,2,3$, are defined by

$$
q_{i}^{a}(s)=\theta_{i}^{l}-\epsilon_{l} \delta_{i}^{a}+\frac{\epsilon_{l}-1}{2}, \quad a \in\left\{a \mid \epsilon_{l} \theta_{a}(l)+\frac{1-\epsilon_{l}}{2}=N_{L}(s)\right\} .
$$

Putting everything together, one finally finds

$$
\begin{aligned}
I_{F T}^{\mathrm{tw}}(F, R, G)=-\frac{i}{2 N} \sum_{k=0}^{N-1} \sum_{l=1}^{N-1} N_{k, l} \epsilon_{l}[ & \operatorname{ch}_{(\mathbf{2 7}, \mathbf{1})}(F) \operatorname{ch}_{\theta_{i}^{l}+\frac{\epsilon_{l}-3}{6}}^{k}(F) \operatorname{ch}_{-\left(1-\theta_{i}^{l}\right)}^{k}(G)+ \\
& \left.+\sum_{s} \operatorname{ch}_{q_{i}(s)}^{k}(F) \operatorname{ch}_{n_{i}(s)}^{k}(G)\right] \widehat{A}(R), \quad(\mathrm{C} .
\end{aligned}
$$

where the sum over the set of oscillator states $s$ now contains also the sum over the

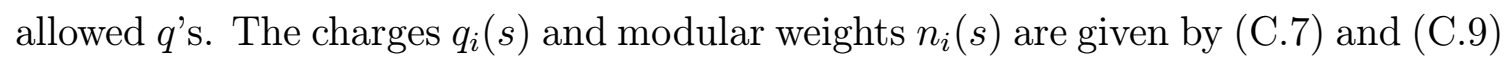
respectively. Observe finally that to each particle $s$ in the $l$-th twisted sector there corresponds an antiparticle $s^{\prime}$ in the $(N-l)$-th conjugate sector with left- and rightmoving oscillators exchanged and opposite charge vector. Since $N_{L}\left(s^{\prime}\right)=N_{L}(s)$, $n_{i}\left(s^{\prime}\right)=1-n_{i}(s)$ and $q_{i}\left(s^{\prime}\right)=-q_{i}(s)$, these two states $s$ and $s^{\prime}$ give identical contributions to the anomaly, as expected. Pairing these contributions, the sums over $l$ can be reduced to $l \in S_{l}$. The sum over $k$ can be done explicitly by noticing that the $k$-dependence hidden in the twisted Chern characters amounts to the phase $\exp 2 \pi k i \sum_{i} v_{i}\left(q_{i}-n_{i}\right)$, which can be checked case by case to be simply 1 . One then obtains eq. (13.2. 


\section{Chiral determinants}

In this appendix, we provide some details about the evaluation of the determinants appearing in section is.; In particular, we show how two different regularizations - modular-invariant and holomorphic - give different results: the elliptic genera $\bar{A}(\tau ; F, R, G)$ and $A(\tau ; F, R, G)$ respectively. The typical quantity to be computed is a chiral determinant with periodicities $\alpha, \beta \in\left[0,1\left[\right.\right.$ and a twist $\lambda:^{10}$

$$
\operatorname{det}_{\alpha, \beta}(\lambda)=\prod_{m, n} \frac{2 \pi}{\tau_{2}}[(m-\beta)-(n+\alpha) \tau+\lambda] .
$$

It is well known that chiral determinants such as (D.D. approach is to multiply (D.Di opposite chirality. However, although the square modulus of (D. it fails to factorize into the product of a holomorphic and an anti-holomorphic parts associated to components with opposite chirality. In order to define in an unambiguous way the original chiral determinant, one can start with $\lambda$ and $\bar{\lambda}$ independent of each other, i.e. different twists in the chiral and antichiral parts, and define the original chiral determinant as

$$
\left.\operatorname{det}_{\alpha, \beta}(\lambda) \equiv \frac{\left|\operatorname{det}_{\alpha, \beta}(\lambda)\right|^{2}}{\operatorname{det}_{\alpha, \beta}(0)}\right|_{\bar{\lambda}=0}
$$

It is worth observing that this definition precisely corresponds to the original form of the determinants yielding the topological generating functional $\bar{A}$. In each spacetime direction there is a bosonic determinant with left twist $\lambda \neq 0$ and right twist $\bar{\lambda}=0$, and a fermionic determinant with $\lambda=0$.

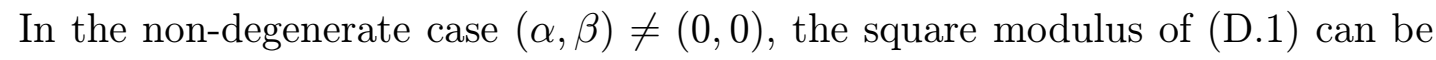

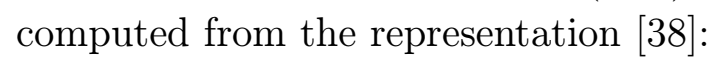

$$
\begin{aligned}
\ln \left|\operatorname{det}_{\alpha, \beta}(\lambda)\right|^{2}=\frac{1}{2} \lim _{s \rightarrow 0} \frac{d}{d s} \sum_{n} & {\left[\int_{C_{+}} d z\left(\frac{e^{i \pi z}}{i \sin \pi z} f_{\alpha, \beta}^{-s}(z, \lambda)-f_{\alpha, \beta}^{-s}(z, \lambda)\right)+\right.} \\
& \left.+\int_{C_{-}} d z\left(\frac{e^{-i \pi z}}{i \sin \pi z} f_{\alpha, \beta}^{-s}(z, \lambda)+f_{\alpha, \beta}^{-s}(z, \lambda)\right)\right],
\end{aligned}
$$

where

$$
f_{\alpha, \beta}(z, \lambda)=\left(\frac{2 \pi}{\tau_{2}}\right)^{2}[(z-\beta)-(n+\alpha) \tau+\lambda][(z-\beta)-(n+\alpha) \bar{\tau}+\bar{\lambda}] .
$$

We have performed a Sommerfeld-Watson transformation to convert the sum over $m$ to an integral over closed contours $C_{ \pm}$following the real axis and going at infinity

\footnotetext{
${ }^{10}$ In our case, the twist $\lambda$ is always a given two-form, so the expressions that will follow are formal and eventually have to be understood as an expansion in $\lambda$.
} 
in the upper/lower half-plane. The first and third terms in the square brackets converge for $s \rightarrow 0$, so that the derivative at $s=0$ can be taken directly. This gives rise to $\ln f_{\alpha, \beta}(z, \lambda)$, which has branch points in the complex plane at the locations $z_{+}=\beta-\lambda+(n+\alpha) \tau$ and $z_{-}=\beta-\bar{\lambda}+(n+\alpha) \bar{\tau}$. The corresponding integrals can then easily be performed, taking care of deforming the $C_{ \pm}$contours to avoid the branch points. Their contribution to $\ln \left|\operatorname{det}_{\alpha, \beta}(\lambda)\right|^{2}$ is

$$
\sum_{n=0}^{\infty} \ln \left|1-q^{n+\alpha} e^{2 \pi i(\beta-\lambda)}\right|^{2}+\sum_{n=1}^{\infty} \ln \left|1-q^{n-\alpha} e^{-2 \pi i(\beta-\lambda)}\right|^{2} .
$$

Let us now consider the second and fourth terms in the square brackets in (D. These terms are convergent only for $\operatorname{Re} s>1 / 2$, so we compute the integrals in this regime and then continue analytically the result to $s=0$. In this case

$$
\begin{aligned}
\int_{C_{+}} d z f_{\alpha, \beta}^{-s}(z, \lambda)-\int_{C_{-}} d z f_{\alpha, \beta}^{-s}(z, \lambda) & =2 \int_{-\infty}^{+\infty} d x f_{\alpha, \beta}^{-s}(x, \lambda) \\
& =2\left[(n+\alpha) \tau_{2}-\operatorname{Im} \lambda\right]^{1-2 s} \frac{\Gamma(1 / 2) \Gamma(s-1 / 2)}{\Gamma(s)} .
\end{aligned}
$$

The sum over $n$ can then be performed using the $\zeta$-function regularization. In this way, one finds a contribution to $\ln \left|\operatorname{det}_{\alpha, \beta}(\lambda)\right|^{2}$ equal to

$$
-2 \pi \tau_{2} B_{2}\left(\alpha-\operatorname{Im} \frac{\lambda}{\tau_{2}}\right)
$$

where $B_{2}(c)=1 / 6+c^{2}-c$ is the second Bernoulli polynomial. ${ }^{11}$ By combining $\left(\overline{\mathrm{D}}_{-}^{-} \overline{7}_{-}\right)$ and $\left(\underline{D} . \mathfrak{D}^{\prime \prime}\right)$, one finally obtains

$$
\begin{aligned}
\left|\operatorname{det}_{\alpha, \beta}(\lambda)\right|^{2}= & e^{-\frac{2 \pi(\operatorname{Im} \lambda)^{2}}{\tau_{2}}} e^{-2 \pi \operatorname{Im} \lambda(1-2 \alpha)}|q|^{1 / 6+\alpha^{2}-\alpha} \times \\
& \times \prod_{n=0}^{\infty}\left|1-q^{n+\alpha} e^{2 \pi i(\beta-\lambda)}\right|^{2} \prod_{n=1}^{\infty}\left|1-q^{n-\alpha} e^{-2 \pi i(\beta-\lambda)}\right|^{2} .
\end{aligned}
$$

As anticipated, this expression fails to factorize into holomorphic and and antiholomorphic parts, but the definition (D.

$$
\operatorname{det}_{\alpha, \beta}(\lambda)=\frac{\theta_{1}\left[\begin{array}{c}
\alpha \\
\beta
\end{array}\right](\lambda \mid \tau)}{\eta(\tau)} e^{\frac{\pi \lambda^{2}}{2 \tau_{2}}}
$$

In the degenerate case $(\alpha, \beta)=(0,0)$, the analysis is similar, but one has to introduce an IR regulator [3. the zero-mode, one then finds

$$
\left|\operatorname{det}_{0,0}^{\prime}(\lambda)\right|^{2}=(2 \pi \lambda)^{-2} e^{-\frac{2 \pi \operatorname{Im} \lambda^{2}}{\tau_{2}}} e^{-2 \pi \operatorname{Im} \lambda}|q|^{1 / 6} \prod_{n=0}^{\infty}\left|1-q^{n} e^{-2 \pi i \lambda}\right|^{2} \prod_{n=1}^{\infty}\left|1-q^{n} e^{2 \pi i \lambda}\right|^{2}
$$

\footnotetext{
${ }^{11}$ Strictly speaking, the result (D. $\left.\bar{D}_{\mathbf{L}}\right)$ is valid only for twists $\alpha<1 / 2$ (for sufficiently small $\lambda$ ). However, by considering the equivalent $(1-\alpha)$-twist the result $\left(\mathbf{I}_{\mathbf{L}} \mathbf{-} \mathbf{7}_{-}^{\prime}\right)$ can actually be extended to all twists $0<\alpha<1$.
} 
and hence

$$
\operatorname{det}_{0,0}^{\prime}(\lambda)=\frac{\theta_{1}(\lambda \mid \tau)}{2 \pi \lambda \eta(\tau)} e^{\frac{\pi \lambda^{2}}{2 \tau_{2}}}
$$

Note that this result for the degenerate case can be verified by an alternative method, which consists in rewriting the determinant in terms of Eisenstein series, for which there is a well-known modular-invariant regularization (see for example [2곔).

The above results for the determinants give rise to the modular-invariant gener-

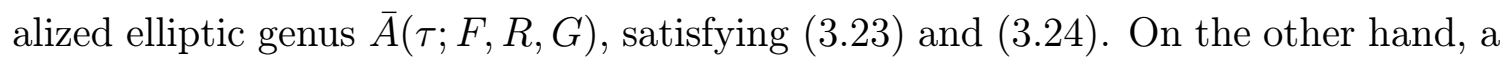
holomorphic regularization can be easily obtained, for example, by defining the effect of the twist $\lambda$ by analytic contribution from the twists $\alpha$ and $\beta$. In other words, the twisted determinant is defined as the value of the ordinary determinant for twist $\left(\alpha^{\prime}, \beta^{\prime}\right)=(\alpha, \beta+\lambda)$. Proceeding in this way, one simply finds the same results as before, (D. $(\overline{8})$ and $\left(\bar{D} . \overline{9}_{i}\right)$, but without the non-holomorphic exponential term. Without this term, modular invariance is lost, but the results are manifestly holomorphic in $\tau$. The resulting elliptic genus is then $A(\tau ; F, R, G)$.

The impossibility of having at the same time holomorphicity and modular invariance is called Quillen's anomaly [3] in heterotic theories.

\section{References}

[1] A. Giveon, M. Porrati and E. Rabinovici, Target space duality in string theory, 'Physys.'

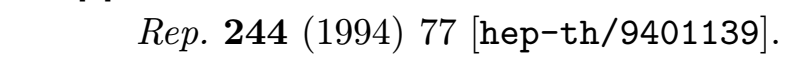

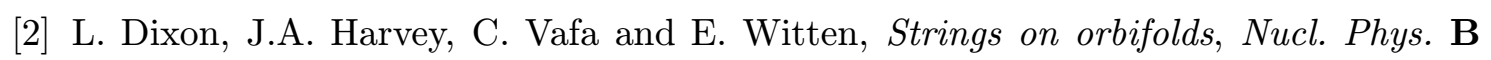
-

[3] L.E. Ibáñez, R. Rabadán and A.M. Uranga, Sigma-model anomalies in compact $D=4$,

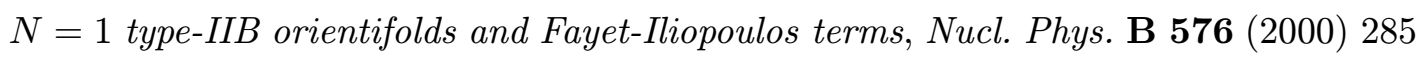
[hep-th/9905098in.

[4] I. Antoniadis, C. Bachas and E. Dudas, Gauge couplings in four-dimensional type-I

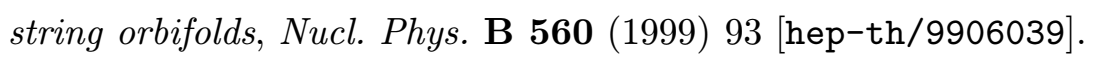

[5] M. Klein, Anomaly cancellation in $D=4, N=1$ orientifolds and linear/chiral multiplet duality, iNucl. Phys. B $5699(2000)$ 362, (hep-th/9910143'.

[6] Z. Lalak, S. Lavignac and H.P. Nilles, Target-space duality in heterotic and type-I

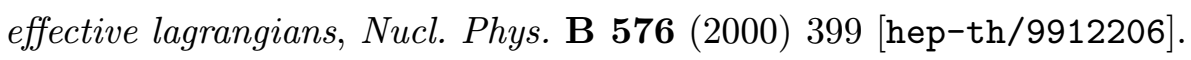

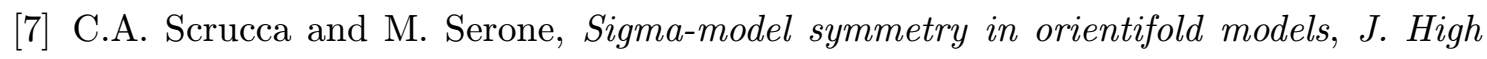

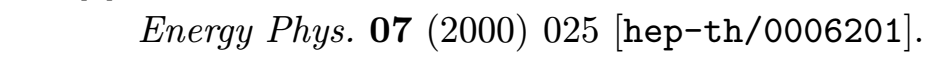

[8] J. Polchinski and E. Witten, Evidence for heterotic-type-I string duality, iNي : - . 
[9] C. Angelantonj, M. Bianchi, G. Pradisi, A. Sagnotti and Y.S. Stanev, Chiral

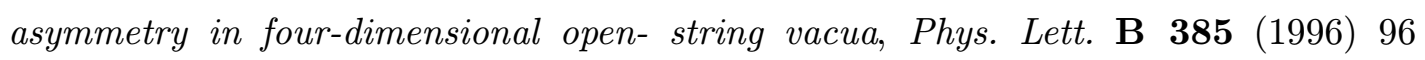
[hep-th/9606169i'.

[10] Z. Kakushadze and G. Shiu, A chiral $N=1$ type-I vacuum in four dimensions and its

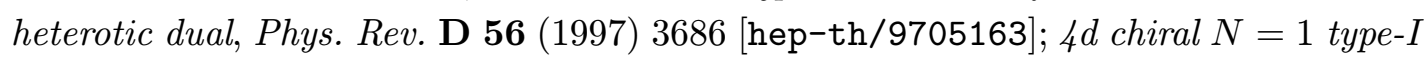
vacua with and without D5-branes, $N$ ucl.

[11] G. Aldazabal, A. Font, L.E. Ibáñez and G. Violero, $D=4, N=1$, type-IIB orientifolds, iNucl. Phys. B 536 (1998) 29: [hep-th/9804026.

[12] Z. Kakushadze, G. Shiu and S.H. H. Tye, Type-IIB orientifolds, F-theory, typeI strings on orbifolds and type-I heterotic duality, $N u c \bar{l}$. [ue. th-

[13] G. Aldazabal, A. Font, L.E. Ibáñez, A.M. Uranga and G. Violero, Non-perturbative

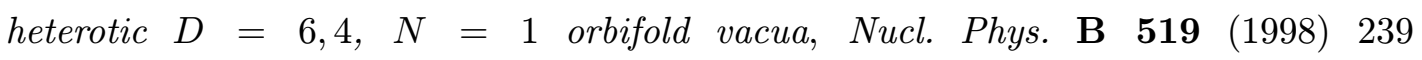
[hep-th/9706158in.

[14] R. Dijkgraaf, E. Verlinde and H. Verlinde, On moduli spaces of conformal field theories with $c \geq 1$, in Copenhagen 1987, Proceedings, Perspectives in string theory, 117.

[15] G. Moore and P. Nelson, Anomalies in nonlinear sigma models, $\bar{P}$ hys. $\bar{R}$ ev. $\bar{L}$ ett. $\overline{\mathbf{5}} \overline{3}_{1}^{\prime}$ :

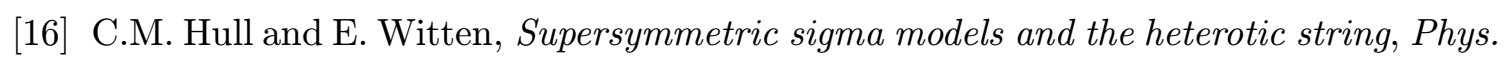
- Lett. B $160-1985)$ 398.

[17] L.J. Dixon, V. Kaplunovsky and J. Louis, Moduli dependence of string loop corrections to gauge coupling constants, №ucl. Phys.

[18] G.L. Cardoso and B.A. Ovrut, A Green-Schwarz mechanism for $D=4, N=1$ supergravity anomalies, №

[19] J.P. Derendinger, S. Ferrara, C. Kounnas and F. Zwirner, On loop corrections to string effective field theories: field dependent gauge couplings and sigma model anomalies, №

[20] L.E. Ibáñez and D. Lüst, Duality anomaly cancellation, minimal string unification

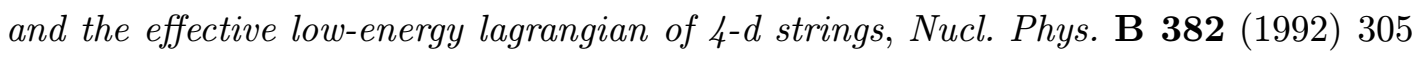
[hep-th/9202046il.

[21] G.L. Cardoso and B.A. Ovrut, Coordinate and Kähler sigma model anomalies and their cancellation in string effective field theories, ${ }_{N}{ }_{-1} u c l$. [hep-th/9205009.'.

[22] M.B. Green and J.H. Schwarz, Anomaly cancellation in supersymmetric $D=10$ gauge

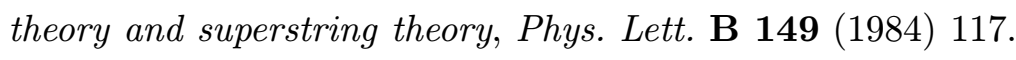


[23] E. Witten, Some properties of $O(32)$ superstrings, ${ }^{2} \bar{h} y s$.

[24] M. Dine, N. Seiberg and E. Witten, Fayet-Iliopoulos terms in string theory, iNuucl: (-

[25] I. Antoniadis, E. Gava, K.S. Narain and T.R. Taylor, Superstring threshold corrections

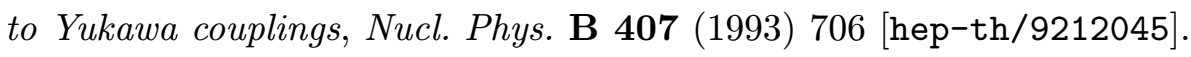

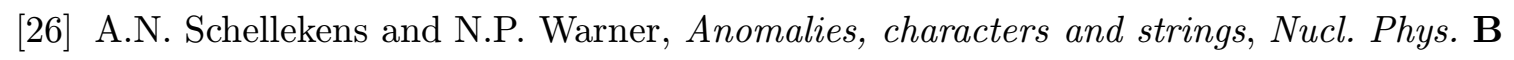
(-

[27] W. Lerche, B.E. W. Nilsson, A.N. Schellekens and N.P. Warner, Anomaly cancelling

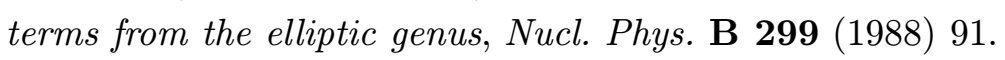

[28] W. Lerche, Elliptic index and superstring effective actions, №cl. Phys. B. - - -

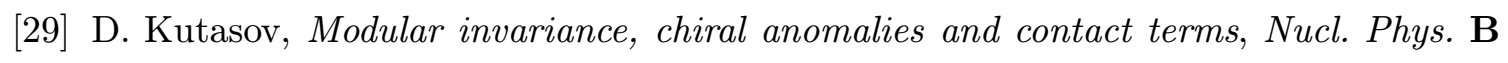
-

[30] H. Suzuki and A. Sugamoto, Role of modular invariance in evaluation of gauge and

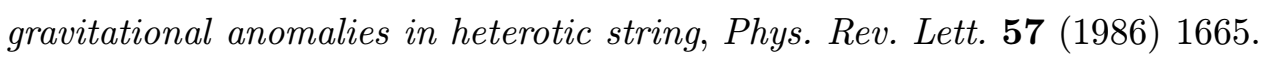

[31] K. Miki, Fermionic strings: zero modes and supermoduli, (-

[32] S. Yahikozawa, Modular invariance of one loop N-point amplitudes in heterotic string theory, Nucl. Phys. B $291-1987) 369$;

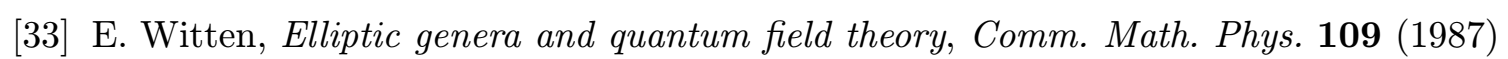
(- -2525 .

[34] C.M. Hull, Compactifications of the heterotic superstring, Phys. -

[35] D. Friedan, E. Martinec and S. Shenker, Conformal invariance, supersymmetry and string theory, iNucl. Phys. B 271 1986$) 93$.

[36] L.J. Dixon, V. Kaplunovsky and J. Louis, On effective field theories describing $(2,2)$ vacua of the heterotic string, ${ }^{1}$ uucl. Phys.

[37] L. Alvarez-Gaumé and E. Witten, Gravitational anomalies, 'Nㅡㄹ. 1. - -

[38] J. Polchinski, Evaluation of the one loop string path integral, (1986) 37 .

[39] D. Quillen, Determinants of Cauchy-Riemann operators over a Riemann surface, Funct. Anal. App. 19 (1985) 31 (English translation from the original Russian edition). 